\title{
Disclosing the Potential of the SARP-Type Regulator PapR2 for the Activation of Antibiotic Gene Clusters in Streptomycetes
}

OPEN ACCESS

Edited by:

Rustam Aminov,

University of Aberdeen,

United Kingdom

Reviewed by:

Marie-Joelle Virolle,

Centre National de la Recherche

Scientifique (CNRS), France

Jean-Luc Pernodet,

Centre National de la Recherche

Scientifique (CNRS), France Javier Santos Aberturas,

John Innes Centre (JIC),

United Kingdom

Juan F. Martin,

Universidad de León, Spain

${ }^{*}$ Correspondence: Yvonne Mast

yvonne.mast@dsmz.de

Specialty section:

This article was submitted to Antimicrobials, Resistance

and Chemotherapy,

a section of the journal

Frontiers in Microbiology

Received: 29 October 2019

Accepted: 30 January 2020

Published: 18 February 2020

Citation:

Krause J, Handayani I, Blin K, Kulik A and Mast Y (2020) Disclosing

the Potential of the SARP-Type

Regulator PapR2 for the Activation

of Antibiotic Gene Clusters

in Streptomycetes.

Front. Microbiol. 11:225.

doi: 10.3389/fmicb.2020.00225
Janina Krause', Ira Handayani, ${ }^{1,2}$ Kai Blin ${ }^{3}$, Andreas Kulik ${ }^{1}$ and Yvonne Mast ${ }^{1,4,5,6 *}$

${ }^{1}$ Department of Microbiology/Biotechnology, Interfaculty Institute of Microbiology and Infection Medicine, Faculty of Science, University of Tübingen, Tübingen, Germany, ${ }^{2}$ Research Center for Biotechnology, Indonesian Institute of Sciences (LIPI), Cibinong, Indonesia, ${ }^{3}$ Novo Nordisk Foundation Center for Biosustainability, Technical University of Denmark, Lyngby, Denmark, ${ }^{4}$ German Center for Infection Research (DZIF), Partner Site Tübingen, Tübingen, Germany, ${ }^{5}$ Department of Bioresources for Bioeconomy and Health Research, Leibniz Institute DSMZ - German Collection of Microorganisms and Cell Cultures, Braunschweig, Germany, ${ }^{6}$ Department of Microbiology, Technical University Braunschweig, Braunschweig, Germany

Streptomyces antibiotic regulatory protein (SARP) family regulators are well-known activators of antibiotic biosynthesis in streptomycetes. The respective genes occur in various types of antibiotic gene clusters encoding, e.g., for polyketides, ribosomally and non-ribosomally synthesized peptides, or $\beta$-lactam antibiotics. We found that overexpression of the SARP-type regulator gene papR2 from Streptomyces pristinaespiralis in Streptomyces lividans leads to the activation of the silent undecylprodigiosin (Red) gene cluster. The activation happens upon the inducing function of PapR2, which takes over the regulatory role of RedD, the latter of which is the intrinsic SARP regulator of Red biosynthesis in S. lividans. Due to the broad abundance of SARP genes in different antibiotic gene clusters of various actinomycetes and the uniform activating principle of the encoded regulators, we suggest that this type of regulator is especially well suited to be used as an initiator of antibiotic biosynthesis in actinomycetes. Here, we report on a SARP-guided strategy to activate antibiotic gene clusters. As a proof of principle, we present the PapR2-driven activation of the amicetin/plicacetin gene cluster in the novel Indonesian strain isolate Streptomyces sp. SHP22-7.

Keywords: actinomycetes, Streptomyces, antibiotic, regulator, SARP, silent gene cluster

\section{INTRODUCTION}

In 2018, the WHO warned that the dramatic increase of antibiotic resistances coupled with the scarcity of new antibiotics will lead to a global health crisis in the 21st century (World Health Organization, 2015). Even nowadays, infections that are caused by drug-resistant pathogens are suggested to account for 700,000 deaths worldwide annually (United Nations Foundation and the Wellcome Charitable Trust, 2016). According to the World Bank Group "by 2050, drug-resistant infections could cause global economic damage on par with the 2008 financial crisis" (Worldbank, 2016). Thus, there is a substantial need for new antibiotics in order to combat drug-resistances. Bacteria have long been recognized as a prolific source for antibiotics 
(Newman and Cragg, 2016). Especially actinomycetes are potent producers of bioactive molecules, as they provide up to $70 \%$ of all medically important antibiotic agents (Tanaka and Omura, 1990). The capability to produce these natural compounds is genetically encoded in the actinomycetes genome, whereby the respective genes usually are organized as biosynthetic gene clusters (BGCs). In recent years, genomic analyses of actinomycetes have revealed the presence of numerous "silent" or "cryptic" BGCs, meaning that these clusters remain silent or are only weakly expressed under standard lab conditions. Indeed it is estimated that actinomycetes encode $\sim 10$-times the number of secondary metabolites than anticipated from prior fermentation studies (Baltz, 2017). Thus, these microorganisms still hold the genetic potential to produce new bioactive compounds. Consequently, there are several attempts to activate silent gene cluster expression in order to find new antibiotics. However, most of these activation efforts are either (a) completely unspecific in terms of the BGC(s) to be activated [e.g., by adding general elicitors to the cell culture, co-cultivation approaches, or strain-cultivations following the "one strain-many compounds" (OSMAC) strategy] or (b) they are absolutely specific for the BGC of interest (e.g., heterologous expression of the BGC, introduction of an artificial promoter in front of the BGC, or manipulation of a cluster-situated regulator) as reviewed in Ochi and Hosaka (2013) and Zhu et al. (2014). Both approaches have their drawbacks, as there are either major analytical efforts to identify the product from the silent gene cluster [in terms of (a)] or tedious genetic engineering efforts to manipulate the producer strain [in terms of (b)]. Thus, it would be highly beneficial to have a more general activation strategy that targets a defined set of BGCs, which would tackle both issues. In a recent study from Martínez-Burgo et al. (2019) it has been shown that conserved pathway-specific activators can be used to activate BGC expression in a foreign Streptomyces strain. In this study the heterologous expression of the PASLuxR type regulator gene pim $M$ from Streptomyces natalensis in Streptomyces clavuligerus led to the activation of clavulanic acid, cephamycin $\mathrm{C}$, and tunicamycin production.

Here we demonstrate that Streptomyces Antibiotic Regulatory Protein (SARP)-type regulators can be used as activators of certain antibiotic gene clusters in actinomycetes and describe a genome-based approach to screen for SARP-activated gene clusters. SARPs have exclusively been found in actinomycetes, especially in streptomycetes, where they act as pathway-specific activators of secondary metabolite biosynthesis (Bibb, 2005). They are known to be associated with various antibiotic gene clusters, encoding type I- (Bate et al., 2002; Takano et al., 2005; Novakova et al., 2011) and type II-PKS derived polyketides (Lombó et al., 1999; Sheldon et al., 2002; Aigle et al., 2005; Novakova et al., 2011), ribosomally (Widdick et al., 2003; Wu et al., 2018) and non-ribosomally synthesized peptides (Ryding et al., 2002), hybrid polyketide-peptide compounds (Pulsawat et al., 2009; Suzuki et al., 2010; Xie et al., 2012; Salehi-Najafabadi et al., 2014; Mast et al., 2015; Ye et al., 2018), $\beta$-azachinones (Santamarta et al., 2002; Rodríguez et al., 2008; Kurniawan et al., 2014), and azoxy compounds (Garg et al., 2002). SARP genes usually are located within the BGC they are regulating. The encoded SARP gene products are characterized by a winged helix-turn-helix (HTH) DNA-binding motif at the $\mathrm{N}$-terminus that binds to a conserved recognition sequence within the major groove of the target DNA (Wietzorrek and Bibb, 1997; Liu et al., 2013). The DNA recognition sequence constitutes direct heptameric repeat sequences followed by $4 \mathrm{bp}$ spacers, which are often localized between the -10 and the -35 promoter element of the respective target DNA. Such a localization has already been described for the SARP type regulator AfsR from Streptomyces coelicolor, which binds to a recognition sequence 8 bp upstream of the -10 element (Tanaka et al., 2007). Also the SARP regulators Aur1PR4 from Streptomyces aureofaciens and FdmR1 from Streptomyces griseus bind to heptameric repeat sequences, which are located 8 bp upstream of the -10 region (Chen et al., 2008; Rehakova et al., 2013). ActII-ORF4 from S. coelicolor interacts with the -35 element for transcriptional activation (Arias et al., 1999). DnrI from Streptomyces peucetius and SanG from Streptomyces ansochromogenes bind to interaction sites that occur within the -35 element (Sheldon et al., 2002; He et al., 2010). It is suggested that in general the SARP binding site overlaps with the -35 region of the target promoter, which is a binding region of the majority of repressors but not activators. Thus, SARPdriven transcriptional activation has been proposed to occur via a novel mechanism (Tanaka et al., 2007). The C-terminal bacterial activation domain (BTAD) of the SARP protein activates the transcription of the target genes by recruiting the RNA polymerase (RNAP) to the respective promoter, where a ternary DNA-SARP-RNAP complex is formed allowing for transcriptional initiation (Tanaka et al., 2007). "Small" SARPtype activators only contain the HTH DNA binding and BTAD domain, whereas "large" SARPS carry additional domains at the C-terminal side of the protein. These domains include a domain of unknown function belonging to the P-loop NTPase family, and one or more copies of a tetratricopeptide repeat (TPR) motif (Liu et al., 2013). A typical "small" SARP-type activator is represented by PapR2, which has been identified as the major activator of pristinamycin biosynthesis in Streptomyces pristinaespiralis (Mast et al., 2015). A papR2 deletion mutant is unable to produce any pristinamycin, depicting that PapR2 is essential for pristinamycin biosynthesis (Mast et al., 2015). In contrast, overexpression of papR2 in $S$. pristinaespiralis leads to an increased pristinamycin production, which shows that PapR2 has an activating function (Mast et al., 2015). With the help of electromobility shift assays (EMSAs) and (quantitative) reverse transcription PCR [RT-(q)PCR] analysis the PapR2 target genes have been identified in the pristinamycin producer and a conserved PapR2 binding site was proposed (Mast et al., 2015).

In this study, we report on the potential of SARP-type regulators as genetic engineering devices for the activation of (silent) BGCs in actinomycetes. SARP-type regulators are present predominantly in actinomycetes with an abundance of $98 \%$ in the genus Streptomyces. We demonstrate that the SARPtype regulator PapR2 activates the silent undecylprodigiosin (Red) gene cluster in Streptomyces lividans. Additionally, we provide evidence for a PapR2-guided activation of a BGC in the poorly studied Indonesian strain isolate Streptomyces sp. 
SHP22-7 (SHP22-7), which yielded an increased production of the nucleoside antibiotic plicacetin.

\section{MATERIALS AND METHODS}

\section{Bacterial Strains, Plasmids, and Cultivation Conditions}

The bacterial strains and plasmids used in this study are listed in Supplementary Table S1. For routine cloning strategies Escherichia coli Novablue (Novagen) was used. S. lividans T7 (Fischer, 1996) and SHP22-7 (Handayani et al., 2018) were applied for antibiotic production analysis, generation of overexpression strains, and transcriptional analysis. Cloning procedures and strain cultivation were carried out as described before (Mast et al., 2015). For cultivation and isolation of RNA, Streptomyces strains were grown in $100 \mathrm{ml}$ of $\mathrm{R} 5$ medium in 500-ml Erlenmeyer flasks (with steel springs) on an orbital shaker $(180 \mathrm{rpm})$ at $28^{\circ} \mathrm{C}$ (Kieser et al., 2000). For isolation of genomic DNA and protoplast transformation experiments, strains were grown in $100 \mathrm{ml}$ of S-medium (Kieser et al., 2000). Kanamycin $(50 \mu \mathrm{g} / \mathrm{ml})$, apramycin $(50 \mu \mathrm{g} / \mathrm{ml})$, or thiostrepton $(20 \mu \mathrm{g} / \mathrm{ml})$ were used for selection when appropriate. For antibiotic production experiments with S. lividans, strains were grown in YEME medium as reported before (Mast et al., 2015). For antibiotic production experiments with SHP22-7, strains (SHP22-7papR2-OE; references: SHP22-7pRM4 and SHP22-7 WT) were grown in $50 \mathrm{ml} \mathrm{NL410} \mathrm{medium} \mathrm{consisting} \mathrm{of} \mathrm{glucose}$ $\left(10 \mathrm{~g} \mathrm{l}^{-1}\right)$, glycerol $\left(10 \mathrm{~g} \mathrm{l}^{-1}\right)$, oat meal $\left(5 \mathrm{~g} \mathrm{l}^{-1}\right)$, soy flour $\left(10 \mathrm{gl}^{-1}\right)$, yeast extract $\left(5 \mathrm{gl}^{-1}\right)$, Bacto casamino acids $\left(5 \mathrm{gl}^{-1}\right)$, $\mathrm{CaCO}_{3}\left(1 \mathrm{~g} \mathrm{l}^{-1}\right)$, and distilled water ( $\mathrm{pH}$ was adjusted to 7.0 with $\mathrm{NaOH}$ ) as a preculture. After 3 days, $10 \mathrm{ml}$ of preculture was transferred to $100 \mathrm{ml}$ of sterile main culture medium NL19, consisting of mannitol $\left(20 \mathrm{gl}^{-1}\right)$, soy flour $\left(20 \mathrm{gl}^{-1}\right)$, and distilled water ( $\mathrm{pH}$ adjusted to $\mathrm{pH} 7.5$ with $\mathrm{NaOH}$ ). Cells were grown for $168 \mathrm{~h}$ at $28^{\circ} \mathrm{C}$.

\section{Molecular Cloning}

Basic procedures for DNA manipulation were performed as described previously (Sambrook et al., 1989; Kieser et al., 2000). Primers used for PCR were obtained from MWG Biotech AG (MWG, Ebersberg, Germany) and are listed in Supplementary Table S1.

\section{Construction of the papR2 Overexpression Strain SHP22-7papR2-OE}

For papR2 overexpression experiments with SHP22-7, the papR2 gene was isolated as a NdeI/HindIII-fragment from plasmid pGM190/papR2 (Mast et al., 2015) and was cloned into the $\mathrm{NdeI} /$ HindIII restriction site of the integrative expression vector pRM4. In the resulting overexpression construct pRM4/papR2, the papR2 gene is under control of the constitutive promoter of the erythromycin resistance gene ermEp*. pRM4/papR2 was transferred to SHP22-7 by protoplast transformation. Transformants were selected with apramycin $(50 \mu \mathrm{g} / \mathrm{ml})$, which resulted in the overexpression strain SHP22-7papR2$O E$. Strain SHP22-7pRM4, harboring the empty pRM4 vector, was generated by protoplast transformation accordingly and served as a reference.

\section{PapR2 Protein Expression in S. lividans}

For papR2 overexpression experiments SLpapR2-OE precultures were grown in $100 \mathrm{ml}$ of YEME liquid medium for 2 days at $28^{\circ} \mathrm{C}$. Five milliliters of preculture was used as inoculum for $100 \mathrm{ml}$ YEME liquid medium as main culture with thiostrepton $(12.5 \mu \mathrm{g} / \mathrm{ml})$ as inductor for gene expression (Mast et al., 2015). The main culture was cultivated for 3 days at $28^{\circ} \mathrm{C}$. PapR2 protein purification was carried out as reported before (Mast et al., 2015).

\section{Spectrophotometrical Analysis for Red Detection}

For Red detection, culture supernatant from SLpapR2-OE and SLPGM190 (reference) was treated as reported in Onaka et al. (2011) and absorption was measured with a Hitachi U-2000 spectrophotometer.

\section{Sample Treatment for SHP22-7 Bioassays and Compound Detection}

For SHP22-7 compound detection and bioassay tests, $5 \mathrm{ml}$ culture samples of SHP22-7papR2-OE (references: SHP22-7pRM4 and SHP22-7 WT) was extracted with $5 \mathrm{ml}$ ethyl acetate for $30 \mathrm{~min}$ at RT. Ethyl acetate samples were concentrated in vacuo completely and then redissolved in $0.75 \mathrm{ml}$ of methanol. Methanolic extracts were used for bioassays and high-performance liquid chromatography/mass spectrometry (HPLC-MS) analysis.

\section{Bioassays}

Antibiotic activity was analyzed in disc diffusion assays using Bacillus subtilis ATCC6051 as test organism. Thirty microliters of methanolic extract from three independent biological samples of SHP22-7 WT, SHP22-7pRM4, and SHP22-7papR2$O E$, respectively, was pipetted on a filter disc, which was placed on a $B$. subtilis test plate. Five microliters of kanamycin $(50 \mu \mathrm{g} / \mathrm{ml})$ was applied as a positive control and $30 \mu \mathrm{l}$ of methanol as negative control to test the functionality of the $B$. subtilis bioassay plates. The plates were incubated overnight at $37^{\circ} \mathrm{C}$. Antibiotic activity was quantified by measuring the diameter of the inhibition zone around the filter discs. The bioassay was carried out as 10 independent biological replicates.

\section{HPLC and HPLC-MS Analysis for Amicetin/Plicacetin Detection}

High-performance liquid chromatography analyses were performed with a HP1090M system with ChemStation 3D software rev. A.08.03 (Agilent Technologies, Waldbronn, Germany) on a Nucleosil C18 column $(5 \mu \mathrm{m}, 125 \mathrm{~mm} \times 3 \mathrm{~mm})$ fitted with a precolumn $(20 \times 3 \mathrm{~mm})$ and with a flow rate of $850 \mu \mathrm{l} \mathrm{min}{ }^{-1}$. Chromatography was done by linear gradient elution from 95.5 solvent $A$ (water with $0.1 \%$ phosphoric acid) to $100 \%$ solvent $B$ [acetonitrile (ACN)] over 15 min. The injection volume was $5 \mu \mathrm{l}$. Multiple wavelength monitoring was 
performed at 210, 230, 260, 280, 310, 435, and $500 \mathrm{~nm}$. UV-Vis spectra were measured from 200 to $600 \mathrm{~nm}$. The evaluation of the chromatograms (210 nm only) was done by means of an in-house HPLC-UV-Vis database.

High-performance liquid chromatography-mass spectrometry analysis of amicetin/plicacetin was performed with an Agilent 1200 series chromatography system (binary pump, high performance autosampler, DAD-detector) coupled with an LC/MSD Ultra Trap System XCT 6330 (Agilent Technologies, Waldbronn, Germany). The sample $(5 \mu \mathrm{l})$ was injected on a Nucleosil 100 C18 column $(3 \mu \mathrm{m}, 100 \times 2 \mathrm{~mm})$ fitted with a precolumn $(3 \mu \mathrm{m}, 10 \times 2 \mathrm{~mm})$ at a flow rate of $400 \mu \mathrm{l} / \mathrm{min}$ and a linear gradient from $100 \%$ solvent $\mathrm{A}(0.1 \% \mathrm{HCOOH}$ in water $)$ to $100 \%$ solvent $\mathrm{B}(0.06 \% \mathrm{HCOOH}$ in $\mathrm{ACN})$ over $15 \mathrm{~min}$ at $40^{\circ} \mathrm{C}$. UV-Vis-detection was done at 220, 260, 280, 360, and $435 \mathrm{~nm}$. Electrospray ionization was performed in positive and negative ultra-scan mode (alternating) with a capillary voltage of $3.5 \mathrm{kV}$ and a drying gas temperature of $350^{\circ} \mathrm{C}$. Detection of $\mathrm{m} / z$ values was conducted with Agilent DataAnalysis for 6300 Series IonTrap LC/MS Version 3.4 (Bruker Daltonik). Upon HPLC-MS analyses amicetin, plicacetin, and plicacetin isomer were identified by comparisons of their UV/visible spectra, retention times, and molecular masses with authentic standards, as $m / z 617.1[\mathrm{M}-\mathrm{H}]^{-}$ and $m / z 516.1[\mathrm{M}-\mathrm{H}]^{-}$, respectively.

\section{AntiSMASH Analysis}

With the webtool antiSMASH whole genomes can be scanned for the occurrence of BGCs. Gene cluster similarity is given in $\%$ and indicates the number of similar genes to a known cluster. Genes are similar if a BLAST-alignment results in an $e$-value $<10^{-5}$ and the sequence identity is $>30 \%$. Additionally, the shortest alignment must enclose $>25 \%$ of the sequence. If all genes of a known cluster can be found in the query cluster, the similarity of the sequences is $100 \%$. The similarity lowers if less genes of the known cluster can be found in the query cluster (Medema et al., 2011).

\section{PatScan Analysis}

PatScan analysis (Blin et al., 2018) was performed with the $S$. lividans T7 genome (GenBank Accession Number ACEY00000000) and the PapR2 consensus sequence $5^{\prime}$-GTCAGSS- $3^{\prime}$ using the software at https: //patscan.secondarymetabolites.org/.

\section{PapR2 Electromobility Shift Assays}

For EMSAs with Red-specific promoter regions, 182 bp DNA fragments of the upstream regions of redP and redQ were amplified by PCR from genomic DNA of S. lividans T7 with primer pairs PredPfw/rv and PredQfw/rv, respectively (Supplementary Table S1). For EMSAs with the pliA promoter region, a 230 bp DNA fragment of the upstream region of pliA was amplified by PCR from genomic DNA of strain SHP22-7 as template and primer pair PpliAfw/rv (Supplementary Table S1). Promoter DNA amplificates included a 16 bp Cy5 adapter sequence, each at the $3^{\prime}$ - and $5^{\prime}$-end, which was added via the respective primer sequences. The generated amplificates were used as templates in a second PCR approach together with a
Cy5 primer (Supplementary Table S1) in order to conduct Cy5 labeling of the promoter regions. Promoter labeling and PapR2 EMSAs were carried out with variable concentrations of PapR2 protein sample as reported before (Mast et al., 2015). To verify the specificity of the PapR2-DNA binding, an excess of unlabeled, specific, and non-specific DNA, respectively, was added to the EMSA mixture as described previously (Mast et al., 2015). DNA bands were visualized by fluorescence imaging using a Typhoon Trio $^{\mathrm{TM}}$ Variable Mode Imager (GE Healthcare).

\section{Transcriptional Analysis by Reverse Transcription Analysis (RT-PCR)}

SLpGM190 and SLpapR2-OE, as well as SHP22-7, SHP22$7 p R M 4$, and SHP22-7papR2-OE were each grown under $p a p R 2-$ overexpression conditions as described above. Thirty milliliters of each cell culture was harvested after $48 \mathrm{~h}$. Cell disruption was carried out with glass beads (150-212 $\mu \mathrm{m}$; Sigma) at 6,500 rpm, $1 \times 20-30 \mathrm{~s}$, using a Precellys Homogenizer (Peqlab). Total RNA was isolated as described previously (Sambrook et al., 1989) and served as the basis for RT-PCR experiments. DNA was removed by digestion with DNase (Thermo Fisher Scientific) and absence of DNA was verified via PCR analysis. RNA concentrations and quality were checked using a NanoDrop ND-1000 spectrophotometer (Thermo Fisher Scientific). cDNA from $3 \mu \mathrm{g}$ RNA was generated with random primers, reverse transcriptase, and cofactors (Fermentas). For RT-PCRs, primers were used that amplify cDNA of 200-250 bp from internal gene sequences. PCR conditions were $98^{\circ} \mathrm{C}$ for $5 \mathrm{~min}$, followed by 30 cycles of $95^{\circ} \mathrm{C}$ for $30 \mathrm{~s}, 60^{\circ} \mathrm{C}$ for $30 \mathrm{~s}$, and $72^{\circ} \mathrm{C}$ for $45 \mathrm{~s}$, and a final cycling step at $72^{\circ} \mathrm{C}$ for $5 \mathrm{~min}$. As a positive control, cDNA was amplified from the $16 \mathrm{~S}$ rRNA transcript, which is transcribed constitutively. To exclude DNA contamination, negative controls were carried out by using total RNA as a template for each RTPCR reaction. At least three independent biological replicates have been tested.

\section{Transcriptional Analysis by Real-Time qPCR}

Real-time qPCR analysis was applied for quantitative cDNA determination. PCR reactions were run with $\mathrm{SYBR}^{\circledR}$ Green Supermix (BioRad) on an iQ5 Multicolor Real-Time-PCR Detection System (BioRad). The $\mathrm{SYBR}^{\circledR}$ Green Dye shows increased fluorescence when bound to double-stranded DNA. The fluorescence is measured at 494 and $521 \mathrm{~nm}$ and gives the proportional amount of generated dsDNA. cDNA was generated from cultures of SLPGM190 and SLPapR2-OE as described above. The primer pairs redPintfw/rv and redQintfw/rv, respectively (Supplementary Table S1), which amplify fragments of about 180 bp from internal gene sequences, were used together with cDNA as template in qPCR reactions. $h r d B$ was used as housekeeping gene in each experiment in order to standardize the results by eliminating variation in RNA and cDNA quantity and quality. Each reaction mixture of $10 \mu \mathrm{L}$ volume contained $5 \mu \mathrm{L}$ SYBR $^{\circledR}$ Green Supermix (BioRad), $3.85 \mu \mathrm{L}$ nuclease-free water, $0.2 \mu \mathrm{L}$ of each primer, and $0.75 \mu \mathrm{L}$ template. PCR conditions were $98^{\circ} \mathrm{C}$ for $5 \mathrm{~min}$, followed by 35 cycles of 
$95^{\circ} \mathrm{C}$ for $20 \mathrm{~s}$, and $57^{\circ} \mathrm{C}$ for $30 \mathrm{~s}$. To determine amplification specificity, melting curve analyses were performed after the last cycle, showing in all cases one single peak. Results were analyzed using the $\Delta \Delta \mathrm{CT}$-method (Livak and Schmittgen, 2001). Changes in gene expression are represented in relation to the data from samples of SLPGM190. Data are presented as the results from six independent biological replicates.

\section{Database Analysis}

FASTA sequences of SARP proteins were extracted from the antiSMASH database version 2 (Blin et al., 2019) by querying for all genes that hit the antiSMASH smCoG (secondary metabolite clusters of orthologous groups) profile SMCOG1041 (transcriptional regulator, SARP family). These hits were checked for the presence of at least one out of four SARP-related profiles from the PFAM database: PF00486.27 (Trans_reg_C, the HTH-style DNA binding domain), PF93704.16 (BTAD, the transcriptional activator), PF00931.22 (NB-ARC, a domain of unknown function found, e.g., in Saccharopolyspora erythraea SARPs), and PF13424.6 (TPR_12, a TPR found in many larger SARPs). Sequences that did not hit at least one of these domains were discarded. Sequences were then annotated by which of the four profiles were hit and grouped by taxonomic order and BCG type.

\section{RESULTS}

\section{PapR2 Induces Expression of the Silent Red Gene Cluster in S. lividans}

Streptomyces lividans is a widely used heterologous host strain, which under specific laboratory conditions does not exhibit production of the two pigmented secondary metabolites actinorhodin and undecylprodigiosin (Hu et al., 2002; Martinez et al., 2005; Rodríguez et al., 2013). In frame of analyzing the regulatory role of PapR2 in S. pristinaespiralis, the papR2 gene was heterologously expressed in S. lividans T7 for protein purification purposes (Mast et al., 2015). For overexpression experiments the $S$. lividans strain SLpapR2-OE was used, which harbors the papR2 gene under control of the thiostrepton-inducible promoter $\mathrm{P}_{\text {tipA }}$ on the replicative medium-copy plasmid pGM190 (Mast et al., 2015). S. lividans strain SLPGM190 was used as a reference, containing the pGM190 empty vector. After 23 days of growth in YEME liquid medium with thiostrepton as inductor for gene expression, the whole SLPapR2-OE culture, as well as the culture supernatant showed an intensive red pigment formation, which was not observed for samples of SLPGM190 (Figure 1A). This phenomenon was also observed on R5 agar with thiostrepton, where SLPapR2-OE mycelium was intensively red colored after 3-4 days of cultivation, whereas SLPGM190 mycelium was not (Supplementary Figure S1). S. lividans is known to harbor a Red BCG, which remains silent under normal growth conditions (Horinouchi et al., 1986). Red biosynthesis has mainly been studied in S. coelicolor; however, since $S$. coelicolor and $S$. lividans are very closely related species, knowledge on Red biosynthesis and regulation can be transferred to S. lividans (van Wezel et al., 2000; Lewis et al., 2010).To investigate, whether the red color of the SLPapR2-OE cultures originates from the formation of the Red metabolite, we performed spectrophotometrical analysis. A pH shift was carried out with the culture supernatant of SLPapR2-OE and the absorption maxima of the sample was determined by using a Hitachi U2000 spectrophotometer. Spectrophotometrical analysis led to the detection of the Red-specific spectral absorption maxima (Onaka et al., 2002) at 533 and $468 \mathrm{~nm}$ under acidic and basic conditions, respectively (Figure 1B), which proved that the SLpapR2-OE samples contained Red (Figure 1C). These data suggested that the overexpression of papR2 in S. lividans induced Red biosynthesis.

\section{PapR2 Mimics the Function of the S. lividans SARP-Type Regulator RedD}

In S. lividans Red biosynthesis is under control of the SARPtype regulator RedD, which directly activates the Red biosynthetic genes (Takano et al., 1992; White and Bibb, 1997). An amino acid sequence comparison using BLASTP revealed that PapR2 and RedD are highly similar to each other (44\% identity, 55\% similarity) (Mast et al., 2015). This amino acid sequence similarity was even higher for the HTH motif of the protein (66\% identity, $75 \%$ similarity). Thus, we suspected that PapR2 may substitute for the function of RedD and activates Red biosynthesis in S. lividans. In order to identify potential SARP-type binding motifs within the S. lividans Red BGC, the genome was analyzed with the bioinformatic tool PatScan, which allows for the identification of specific sequence patterns in a given genome sequence (Blin et al., 2018). PatScan analysis was performed with the previously described PapR2 consensus motif (5'-GTCAGSS-3') (Mast et al., 2015) as sequence pattern on the $S$. lividans genome sequence. Thereby, two highly conserved PapR2-like motifs were identified within the intergenic region of the Red-specific biosynthetic genes redP (SCO5888) and redQ (SCO5887) with each 100 and 96.5\% identity, respectively (Figure $2 \mathrm{~A}$ ). redP encodes a 3ketoacyl-acyl carrier protein synthase, whereas redQ codes for an acyl carrier protein, both of which have been shown to be involved in Red biosynthesis (Mo et al., 2008). To analyze the functionality of the identified SARP motifs, EMSAs were performed with the PapR2 protein and the upstream regions of redP (PredP, 182 bp) and redQ (PredQ, 182 bp), respectively, harboring the PapR2 consensus sequence. EMSAs showed that PapR2 specifically binds to the PredP and PredQ fragment, respectively (Figure $2 \mathrm{~B}$ ), revealing the functionality of the identified SARP-type motifs.

To confirm the regulatory effect of PapR2 on the transcription of the Red BGC, we performed RT-PCR and quantitative qPCR experiments. For these studies SLPapR2-OE, as well as the reference strain SLPGM190 were grown in R5 medium. After $72 \mathrm{~h}$ of cultivation samples were harvested for RNA isolation. For each strain six biological replicates were performed. Isolated RNA was used as a template in RT-PCR experiments as a negative control (Figure 3A), whereas cDNA was used together with 16S primers as a positive control (Figure 3B). For red gene-specific transcriptional analysis, isolated RNA was used as template for RT-PCR experiments with the primer pairs redPintfw/rv 


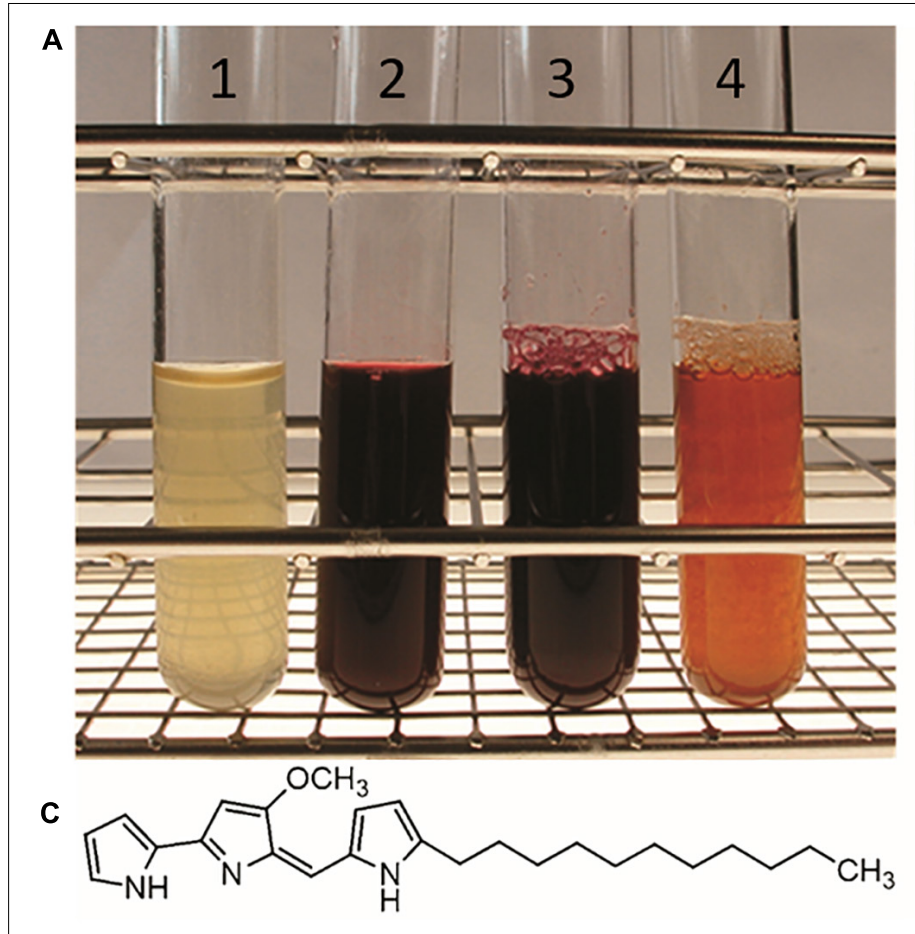

B

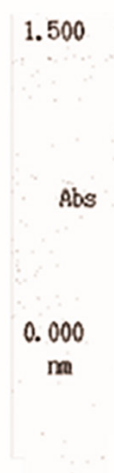

1.500

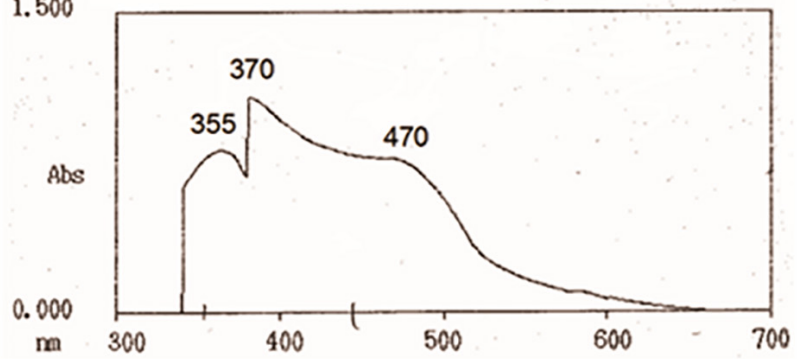

FIGURE 1 | Culture filtrates from SLpGM190 (1) and SLpapR2-OE (2) samples after 3 days of cultivation. Culture filtrate of SLpapR2-OE under acidic (3) and basic (4) conditions (A). Absorption profile of the SLpapR2-OE culture filtrate under acidic (upper graph) and basic condition (lower graph) (B). Chemical structure of Red (C).

and redQintfw/rv, which annealed to internal parts of redP and redQ, respectively. The transcriptional analysis revealed that there are stronger signals for the redP and redQ CDNA amplificates in SLPapR2-OE samples compared to SLPGM190 samples (Figures 3C,D, respectively). In order to quantify the amount of redP and redQ RT-qPCR was performed. Based on the threshold cycle it was calculated that redP and redQ transcripts were increased to 57- and 492-fold, respectively, in samples of SLpapR2-OE compared to samples of SLPGM190 (Supplementary Figure S2). Thus, transcriptional analyses demonstrated that PapR2 activates the transcription of the Red biosynthetic genes redP and redQ. Due to the intensive Red production of strain SLPapR2-OE together with the data obtained from PapR2 EMSA studies and transcriptional analysis, we propose that overexpressed PapR2 in S. lividans takes over the regulatory function of RedD and activates the transcription of the Red biosynthetic genes, which leads to the formation of the red colored secondary metabolite Red.

\section{PapR2 Expression Improves Antibiotic Activity of SHP22-7}

In order to study the general application of SARP-type regulators for activation of antibiotic biosynthesis, we exemplary tested strain SHP22-7 as a host for papR2 expression. SHP22-7 is a novel strain isolate from a soil sample of the unique desert island Enggano, Indonesia (Handayani et al., 2018). SHP22-7 shows broad-spectrum antibacterial activity against Gram-positive and Gram-negative bacteria, including E. coli,
Staphylococcus carnosus, Micrococcus luteus, and B. subtilis. The SHP22-7 genome has been sequenced recently and antiSMASH analysis led to the identification of 25 potential secondary metabolite gene clusters (Handayani et al., 2018; Table 1). Four of the gene clusters (clusters 3,6,10, and 15) contain SARP genes, which makes SHP22-7 a good candidate strain for a SARPguided activation. For papR2 expression studies in SHP22-7, the $p a p R 2$ gene was cloned into the integrative plasmid pRM4 under control of the constitutive $e r m E^{*}$ promoter, resulting in construct $\mathrm{pRM} 4 / \mathrm{papR} 2$. The $\mathrm{pRM} 4$ vector was used as expression plasmid in these analyses to avoid addition of thiostrepton as inductor (see above), which would influence subsequent antibacterial bioassays. The plasmid was transferred to SHP227 by protoplast transformation. The resulting expression strain SHP22-7papR2-OE, as well as the two reference strains SHP22$7 p R M 4$, which harbors the empty pRM4 vector, and the SHP22-7 wild-type (WT) were used for antibacterial bioassay studies. SHP22-7papR2-OE, SHP22-7pRM4, and SHP22-7 WT were each cultivated in NL19 medium and samples were taken at $168 \mathrm{~h}$. Methanolic culture extracts were applied for antibacterial bioassays using $B$. subtilis as test organism. Filter discs with kanamycin and methanol served as positive and negative control, respectively. Bioassays were carried out as 10 independent biological replicates. Thereby, significantly larger inhibition zones against $B$. subtilis were observed on average with extracts from SHP22-7papR2-OE compared to extracts from SHP22$7 p R M 4$ or SHP22-7 WT (Figure 4A). The extract of SHP22$7 p a p R 2-O E$ caused a zone of inhibition of $11.7 \pm 2.1 \mathrm{~mm}$, whereas the extracts of SHP22-7pRM4 and SHP22-7 WT yielded smaller 


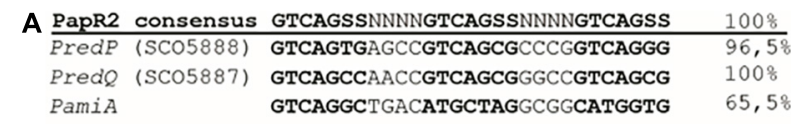

B

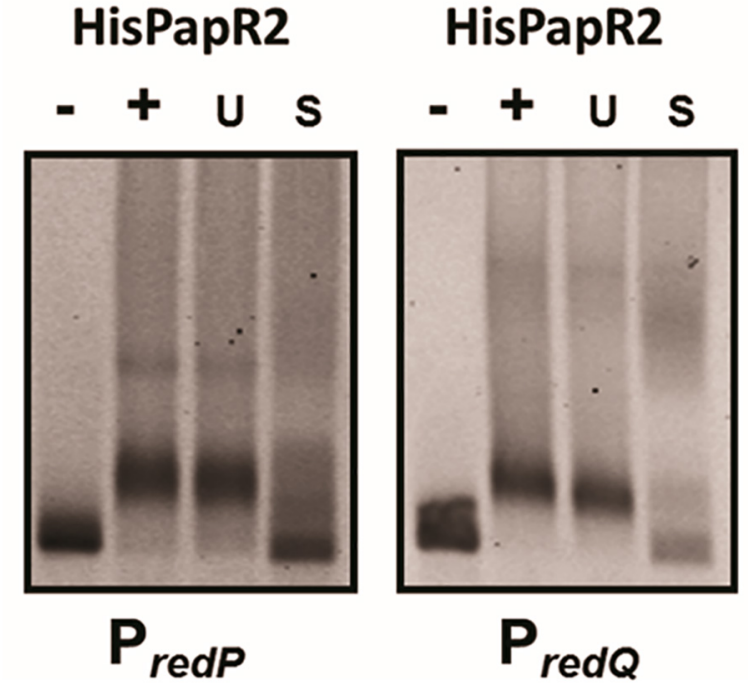

FIGURE 2 | SARP binding sequences within the upstream region of the RED-biosynthetic genes redP (SCO5888), redQ (SCO5887), and pliA (A) EMSAs with undefined HisPapR2 concentrations and Cy5-labeled promoter regions of redP and redQ. - indicates negative control without protein, + indicates addition of HisPapR2 protein. The specificity of the reaction was checked by the addition of 500 -fold specific (S) and unspecific (U) unlabeled DNA (B).

inhibitions zones of $8.3 \pm 2.6$ and $7.3 \pm 2.7 \mathrm{~mm}$, respectively (Figure 4B). These data showed that papR2 expression in SHP227 leads to a significantly improved antibiotic activity.

\section{PapR2 Activates Transcription of Cluster 9 in SHP22-7}

To identify the substance that is responsible for the antibiotic activity of SHP22-7 but avoid elaborative chemical analytics in the first place, we screened the SHP22-7 genome sequence for the occurrence of PapR2-like consensus sequences by using the PatScan tool. Here, we only considered motifs that were located within intergenic regions of genes from suggested SHP227 BGCs. The motif search led to the identification of PapR2like consensus sequences within 5 of the 25 predicted BGCs from SHP22-7. These included clusters 6 and 10 (NRPS-like gene cluster), cluster 15 (type 2 PKS-butyrolactone-like gene cluster), as well as clusters 3 and 9 ("other" type of gene cluster) (Table 1). In order to find out which of the five gene clusters is activated by PapR2, comparative RT-PCR analysis was carried out with SHP22-7papR2-OE, SHP22-7pRM4, and SHP22-7 WT in four independent biological replicates. Cells were grown under antibiotic production conditions and samples were harvested at $168 \mathrm{~h}$ of cultivation. Two samples were collected for each strain and growth time point, whereby one sample was used for RT-PCR analysis and the second one for HPLCMS analysis (see the section "SARP-Type Regulatory Genes Are
Widespread in BGCs From Diverse Actinobacteria"). RT-PCR was carried out with RNA isolated from cell pellets of SHP227papR2-OE, SHP22-7pRM4, and SHP22-7 WT, respectively. Isolated RNA was used as a template in RT-PCR experiments as a negative control (Figure 5A), whereas cDNA was used together with $16 \mathrm{~S}$ primers as a positive control (Figure 5B). For cluster specific transcriptional analysis, cDNA was generated with primer pairs cl3fw/rv, cl6fw/rv, cl9fw/rv, cl10fw/rv, and cl15fw/rv (Supplementary Table S1), each aligning to a predicted biosynthesis gene of clusters $3,6,9,10$, and 15, respectively. The transcriptional analysis revealed that there is a stronger signal for the cluster 9 amplificate in the SHP22-7papR2-OE samples compared to the samples of SHP22-7pRM4 and SHP22-7 WT, where there is nearly no signal at all (Figure 5C). This difference in signal intensity could not be observed for clusters $3,6,10$, and 15 (Figure 5C). Thus, these data suggested that PapR2 activates the transcription of cluster 9 in SHP22-7papR2-OE.

\section{Cluster 9 Resembles an Amicetin Gene Cluster}

In order to deduce for what type of metabolite cluster 9 encodes for, we analyzed the cluster sequence region in detail. The antiSMASH output predicted a $75 \%$ similarity of cluster 9 to the plicacetin/amicetin gene cluster from Streptomyces vinaceusdrappus NRRL 2363 (Table 1). The cluster similarity describes the number of genes with a similarity above the ClusterBlast threshold of $30 \%$ sequence similarity at over $25 \%$ coverage. Manually cluster analysis by sequence alignments yielded a $100 \%$ sequence similarity since the sequence region from $a m i A-a m i D$ was not recognized by antiSMASH to be part of the amicetin BGC (Figures 6A vs. B). Plicacetin (Figure 6C) and amicetin (Figure 6D) are disaccharide pyrimidine nucleoside antibiotics with a broad-spectrum antibacterial (especially against Mycobacterium tuberculosis) and antiviral activity. They act as peptidyl transferase inhibitors and thus inhibit protein synthesis. Amicetin consists of the two deoxysugar moieties, D-amosamine and D-amicetose, as well as cytosine, $p$-aminobenzoic acid (PABA), and a terminal methylserine moiety, whereby the latter moiety is missing in plicacetin (Zhang G.G. et al., 2012; Korzybski et al., 2013). The gene cluster analysis of SHP22-7 revealed that the PapR2-like motif is located directly in front of the orf pliA (Figure 6B) and shows a rather weak sequence identity of $65.5 \%$ to the PapR2 consensus motif (Figure 2A). The same motif is present upstream of amiA in S. vinaceusdrappus. The pliA gene presents $99.75 \%$ gene nucleotide sequence identity to amiA of $S$. vinaceusdrappus, which translates to a $100 \%$ amino acid sequence identity among the predicted gene products. In $S$. vinaceusdrappus amiA is the first gene of the amicetin BCG and encodes a putative 4-amino-4-deoxychorismate lyase, which is suggested to catalyze the conversion from 4-amino-4deoxychorismate to PABA (Zhang G.G. et al., 2012). Remarkably, all genes in the amicetin gene cluster of $S$. vinaceusdrappus and the amicetin-like gene cluster of SHP22-7 are organized in one direction, suggesting a unidirectional transcription. In this context, it would make sense that regulatory activation targets the promoter of the first gene of the unidirectional BGC. In order to 
A

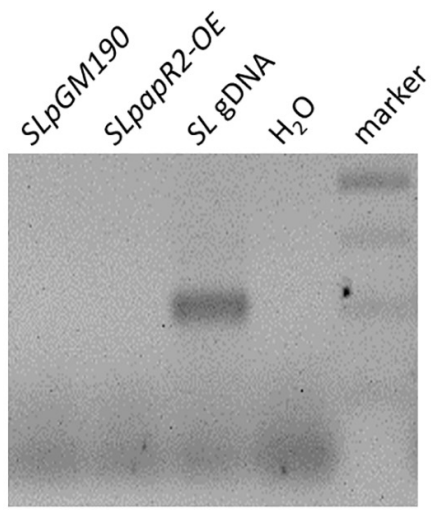

RNA, 16S control

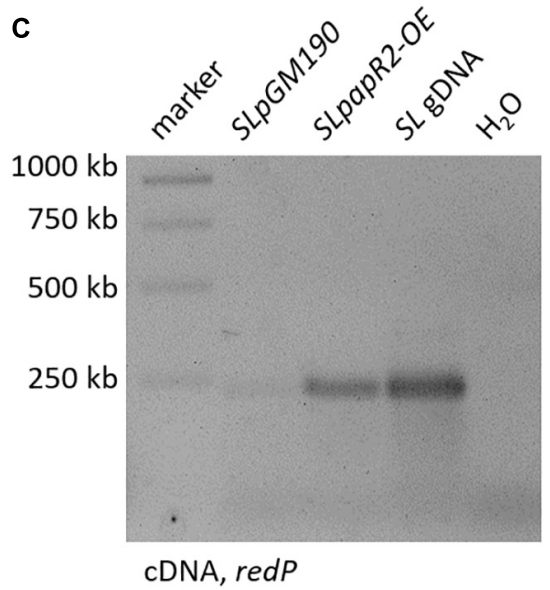

B

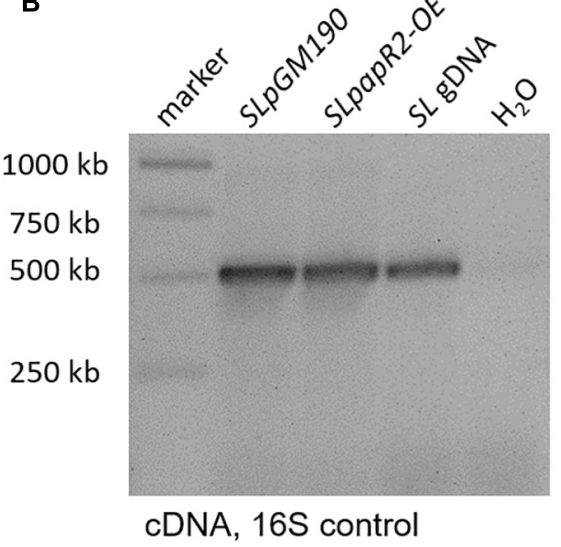

D

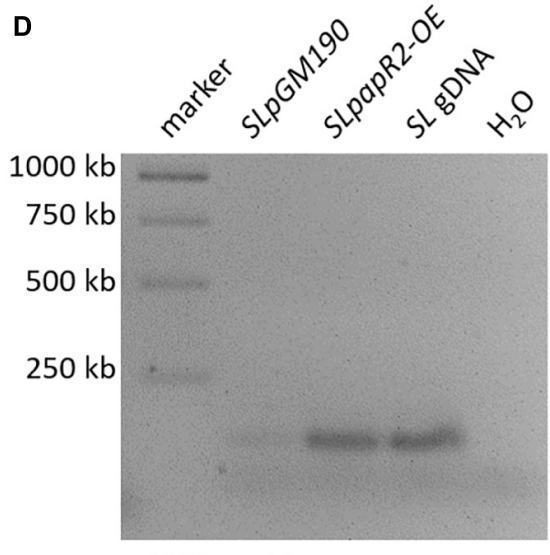

cDNA, redQ

FIGURE 3 | Transcriptional analysis of samples from SLpGM190 and SLpapR2-OE after 168 h. gDNA of S. lividans was used as internal positive control (SL gDNA) and water as internal negative control. RT-PCR analysis with RNA samples as template and 16S-specific primers in order to test the absence of DNA (A). RT-PCR analysis with cDNA samples as template and 16S-specific primers in order to test for successful cDNA synthesis (B). RT-PCR analysis of the gene redP and in the strains SLpGM190 and SLpapR2-OE (C). RT-PCR analysis of the gene redQ and in the strains SLpGM190 and SLpapR2-OE (D).

find out if PapR2 can bind to the pliA promoter region, EMSAs were performed with the PapR2 protein and the pliA upstream region containing the PapR2 consensus sequence. However, no shifted band was detected in these assays (data not shown). Thus, it might be that the motif is not functional at all and does not constitute a SARP binding motif. It could also be that due to the less conserved PapR2 consensus sequence, the motif is not functional in such an in vitro assay. The presence of a SARP consensus sequence would hint for a pathway-specific regulatory gene located within the amicetin(-like) BGC. However, a SARPtype regulatory gene has not been identified in any of the amicetin(-like) clusters from SHP22-7 nor S. vinaceusdrappus (Table 1; Zhang X. et al., 2012). Overall, six SARP genes have been identified in total within the SHP22-7 genome (Supplementary Table S2). All of them are part of BGCs (clusters 3, 6, 10, and 15). Cluster 15 harbors three SARP genes, whereas the other BGCs each contain one SARP gene (Table 1). Furthermore, putative SARP binding motifs have been found within the promoter regions of the BGCs (Table 1 and Supplementary Table S2).
It might be possible that one of these SARP-type regulators plays a role in trans-activating amicetin cluster transcription. So far, regulation of amicetin biosynthesis is not understood. In $S$. vinaceusdrappus three genes $[\operatorname{orf}(-3), \operatorname{orf}(-2), \operatorname{ami} P]$ have been identified, which encode for putative transcriptional regulators, whereby $\operatorname{orf}(-3)$ and $\operatorname{orf}(-2)$ seem not to be part of the amicetin BGC and amiP codes for TetR-like transcriptional regulator, which usually function as repressors of antibiotic biosynthesis (Zhang G.G. et al., 2012). Thus far, it cannot be excluded that also trans-acting regulator(s) are involved in the regulation of amicetin biosynthesis.

\section{PapR2 Activates Plicacetin Production in SHP22-7}

To investigate if an amicetin(-like) antibiotic is produced by SHP22-7, we analyzed methanolic extracts of cell culture samples from SHP22-7papR2-OE by HPLC/MS. To analyze if production is influenced by $p a p R 2$ expression, extracts from samples of 
TABLE 1 | Secondary metabolite gene clusters of Streptomyces sp. SHP22-7 as predicted by antiSMASH 4.0 with an indication of the presence of cluster-situated SARP genes (count), predicted SARP proteins with amino acid sequence homology to PapR2 ( $=$ identity, $\mathrm{S}=$ similarity), as well as identified SARP-type motifs.

\begin{tabular}{|c|c|c|c|c|c|c|}
\hline Cluster & $\begin{array}{l}\text { Type of secondary } \\
\text { metabolite gene cluster }\end{array}$ & $\begin{array}{l}\text { Similarity to known } \\
\text { cluster }\end{array}$ & Localization & $\begin{array}{l}\text { Cluster-situated } \\
\text { SARP gene }\end{array}$ & $\begin{array}{c}\text { Sequence homology } \\
\text { to PapR2 I/S }\end{array}$ & $\begin{array}{l}\text { SARP consensus } \\
\text { motif(s) identified }\end{array}$ \\
\hline 1 & Terpene & Albaflavenone (100\%) & Contig 1 49588-70841 & - & & - \\
\hline 2 & T2PKS & Spore pigment (66\%) & Contig 1 121312-163740 & - & & - \\
\hline 3 & Other & Granaticin (8\%) & Contig 2 304806-348126 & $+(1)$ & $41 / 52 \%$ & + \\
\hline 4 & Melanin & Melanin (60\%) & Contig 3 1-5936 & - & & - \\
\hline 5 & Ectoine & Ectoine (100\%) & Contig 4 119873-130271 & - & & - \\
\hline 6 & NRPS & $\begin{array}{l}\text { Phosphonoglycans } \\
(5 \%)\end{array}$ & Contig 5 129226-170626 & $+(1)$ & $31 / 44 \%$ & + \\
\hline 7 & Bacteriocin & - & Contig 7 213837-225168 & - & & - \\
\hline 8 & Terpene & - & Contig 7 240589-262769 & - & & - \\
\hline 9 & Other & Amicetin (75\%) & Contig $10129625-170245$ & - & & + \\
\hline 10 & NRPS & $\begin{array}{l}\text { Calcium dependent } \\
\text { antibiotic }(47 \%)\end{array}$ & Contig 10 243082-272180 & $+(1)$ & $38 / 56 \%$ & + \\
\hline 11 & T3PKS & Herboxidiene (8\%) & Contig $1120911-62017$ & - & & - \\
\hline 12 & Siderophore & $\begin{array}{l}\text { Desferrioxamine B } \\
(100 \%)\end{array}$ & Contig 13 127488-139260 & - & & - \\
\hline 13 & Indole & Antimycin (20\%) & Contig 17 55086-76126 & - & & - \\
\hline 14 & Terpene & Carotenoid (36\%) & Contig 17 133951-147999 & - & & - \\
\hline 15 & T2PKS-Butyrolactone & Fluostatin (26\%) & Contig 19 32007-96281 & $+(3)$ & $\begin{array}{c}41 / 59 \% 67 / 76 \% \\
40 / 51 \%\end{array}$ & + \\
\hline 16 & Terpene & Hopene (84\%) & Contig 20 8526-35239 & - & & - \\
\hline 17 & Lanthipeptide & - & Contig 21 82417-98812 & - & & - \\
\hline 18 & T1PKS-NRPS & Candicidin (90\%) & Contig 22 1-86799 & - & & - \\
\hline 19 & Arylpolyene-NRPS & Lipopeptide (29\%) & Contig 24 1-40688 & - & & - \\
\hline 20 & Siderophore & Grincamycin (8\%) & Contig 24 46763-57062 & - & & - \\
\hline 21 & T1PKS & Oligomycin (44\%) & Contig 32 4049-49007 & - & & - \\
\hline 22 & OtherKS & Sanglifehrin A (13\%) & Contig 42 1-35910 & - & & - \\
\hline 23 & Bacteriocin & Informatipeptin (57\%) & Contig 44 22689-32904 & - & & - \\
\hline 24 & NRPS & Coelichelin (27\%) & Contig 59 1-16803 & - & & - \\
\hline 25 & Lanthipeptide & - & Contig 96 1-5597 & - & & - \\
\hline
\end{tabular}

+, cluster contains gene/motif; -, cluster does not contain gene/motif.
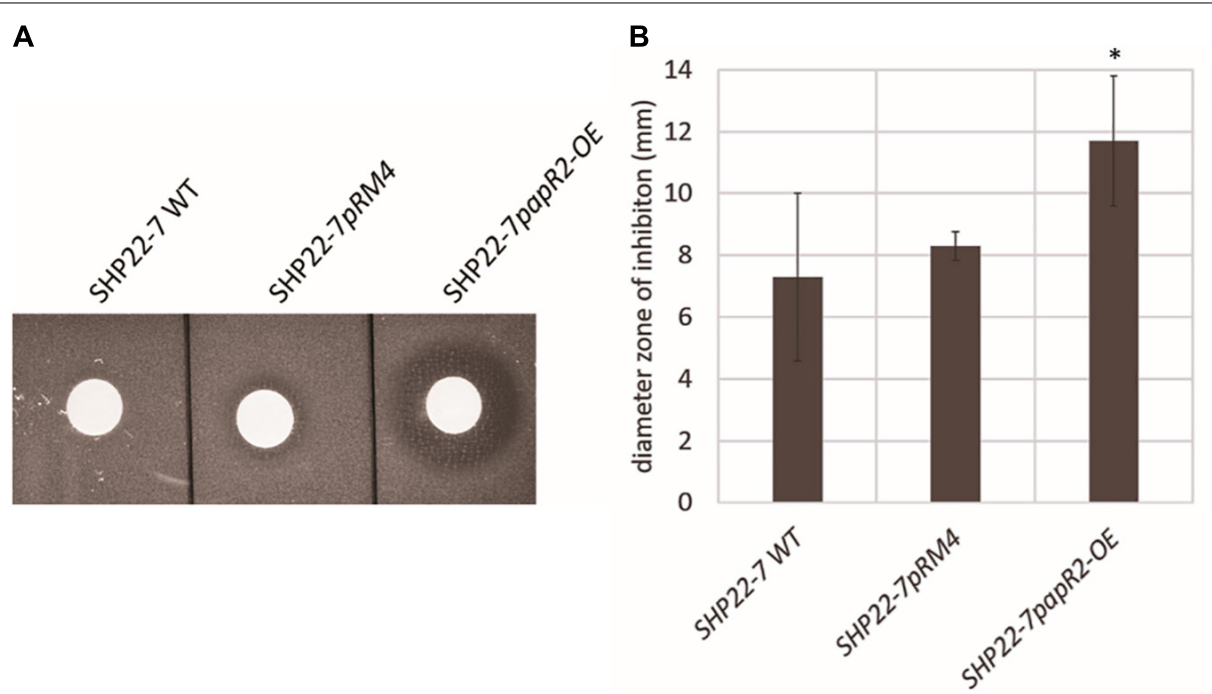

FIGURE 4 | One representative example of culture extracts from SHP22-7 WT, SHP22-7pRM4, and SHP22-7papR2-OE, respectively, against B. subtilis. Filter disk diffusion assay; red arrow indicates inhibition zone (A). Graphical representation of the inhibition zone diameters ( $\mathrm{mm}$ ) from 10 independent biological replicates $(n=10),{ }^{*}$ indicates significance (B). 


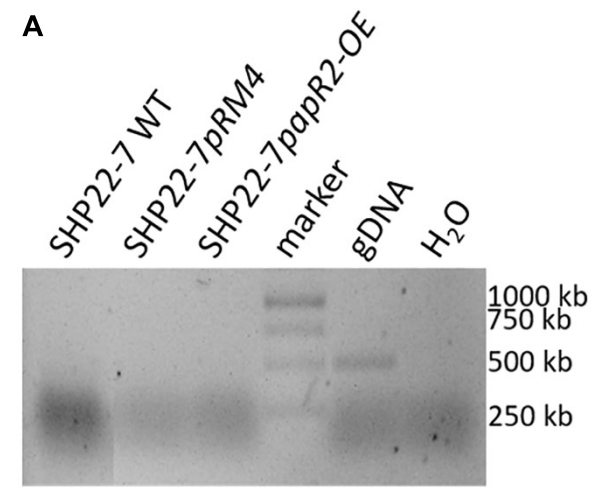

RNA, 16S control
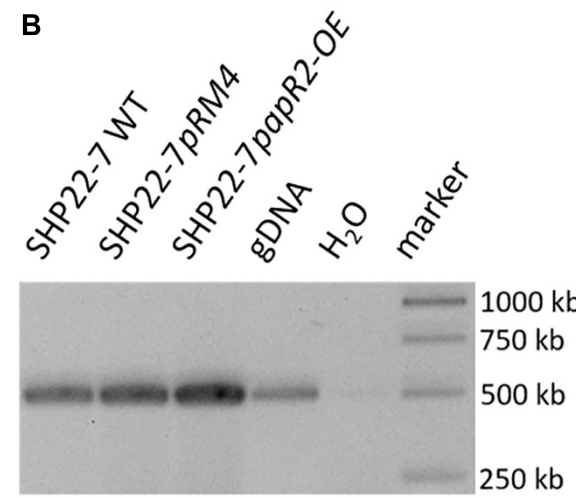

cDNA, $16 \mathrm{~S}$ control
C

cl. 3
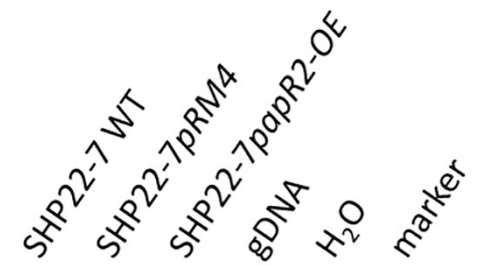

$1000 \mathrm{~kb}$

$750 \mathrm{~kb}$

$500 \mathrm{~kb}$

$250 \mathrm{~kb}$

$1000 \mathrm{~kb}$

$750 \mathrm{~kb}$

$500 \mathrm{~kb}$

cl. 6

$250 \mathrm{~kb}$

$1000 \mathrm{~kb}$

$750 \mathrm{~kb}$

$500 \mathrm{~kb}$

cl. 9

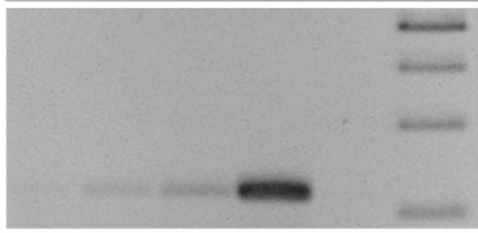

$250 \mathrm{~kb}$

$1000 \mathrm{~kb}$

$750 \mathrm{~kb}$

$500 \mathrm{~kb}$

cl. 10

$250 \mathrm{~kb}$

$1000 \mathrm{~kb}$

$750 \mathrm{~kb}$

$500 \mathrm{~kb}$

cl. 15

$=$

$250 \mathrm{~kb}$

FIGURE 5 | Transcriptional analysis of the SHP22-7 strains SHP22-7 WT, SHP22-7pRM4, and SHP22-7papR2; gDNA of SHP22-7 was used as internal positive (gDNA) and water as internal negative control. The data are representative for four independent experiments. RT-PCR analysis with RNA as template and 16S-specific primers (A). RT-PCR analysis with cDNA and 16S-specific primers (B). Transcriptional analysis of the SARP motif containing gene clusters (cl.) 3, 6, 9 , 10, and 15 with samples from SHP22-7 WT, SHP22-7pRM4, and SHP22-7papR2 (C).

SHP22-7pRM4 and SHP22-7 WT served as references. For these analyses we used the second culture samples obtained from SHP22-7papR2-OE, SHP22-7pRM4, and SHP22-7 WT, respectively (see above). Based on comparisons with an inhouse substance database, HPLC analysis revealed the presence of amicetin [retention time (RT) $4.0 \mathrm{~min}$ ], plicacetin (RT $4.8 \mathrm{~min}$ ), and a plicacetin isomer (RT $5.6 \mathrm{~min}$ ) in all three samples (Figure 7A). The identity of the compounds was verified by MS/MS analysis (amicetin $m / z 617.1$ [M-H]; plicacetin $\mathrm{m} / z 516.1$ [M-H]; plicacetin isomer $m / z 516.1[\mathrm{M}-\mathrm{H}]$ ) (Figure 7B; for HRMS data see Supplementary Figures S3, S4). Comparisons between the HPLC spectra from samples of SHP22-7papR2-OE, SHP22-7pRM4, and SHP22-7 WT displayed that peak intensities were especially increased for plicacetin (mAU 605) and the plicacetin isomer (mAU 259) in samples of SHP22-7papR2-OE compared to samples of SHP22-7pRM4 [plicacetin (mAU 120), plicacetin isomer (mAU 97)] and SHP22-7 WT [plicacetin (mAU 66), plicacetin isomer (mAU 57)] (Figure 7C). Thus, it could be shown that papR2 expression in SHP22-7 activates explicitly plicacetin biosynthesis, whereas amicetin biosynthesis seems not to be affected. Overall, with this result it could be confirmed that PapR2 induced cluster 9 transcription, which resulted in an increased plicacetin production. Thus, it can be concluded that the increased bioactivity of SHP22-7papR2-OE samples against $B$. subtilis arises from the increased production of the nucleoside antibiotic plicacetin. However, since no direct interaction of the PapR2 regulator with the amicetin promoter region could be shown by EMSA analysis, it cannot be deduced if the activation effect is a direct or an indirect one. The SARP consensus motif harbors the central TCA triad, which is also present in the Pho 


\section{A S. vinaceusdrappus}

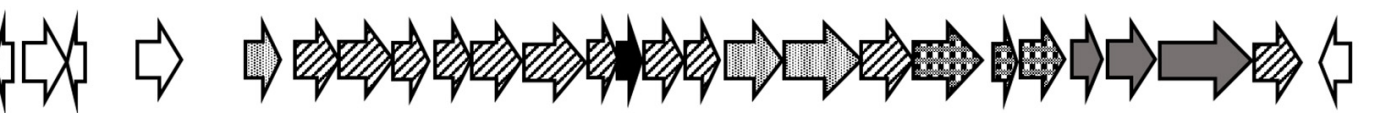

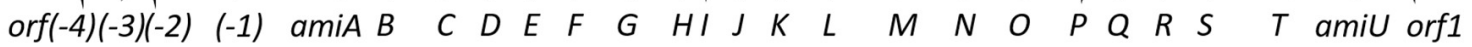

B $\quad$ SHP22-7 SARP consensus
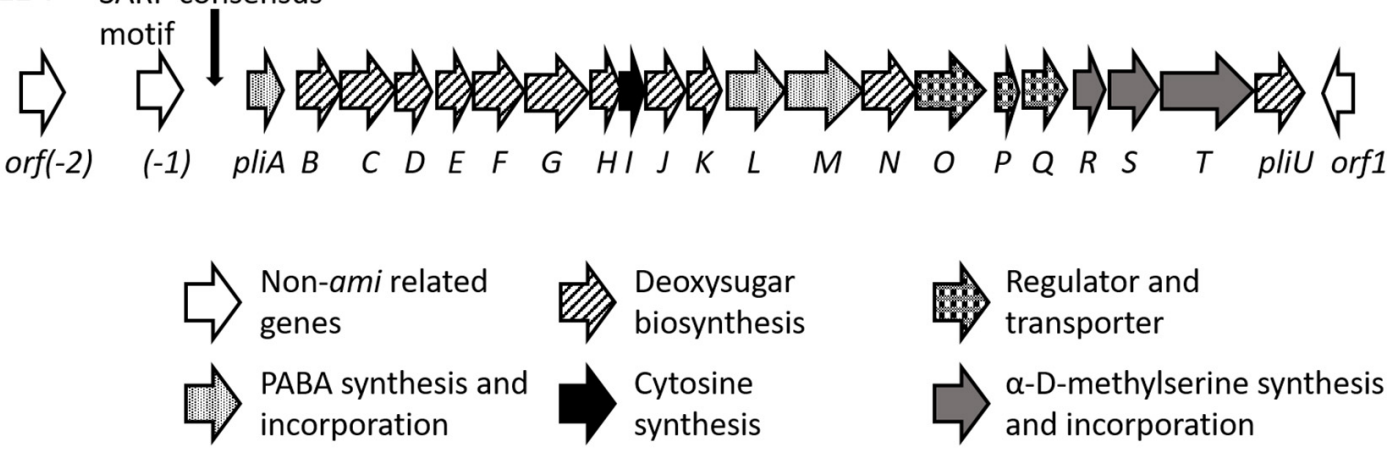

C

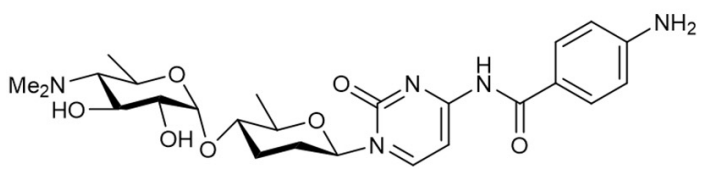

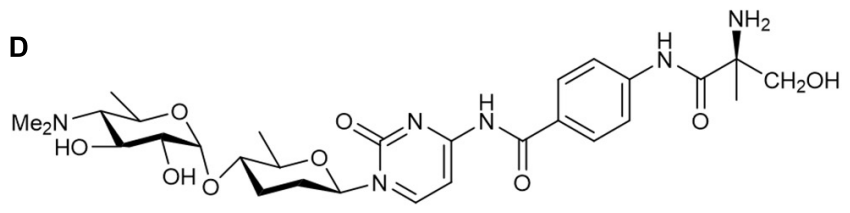

FIGURE 6 | Schematic organization of the amicetin biosynthetic gene cluster of S. vinaceusdrappus according to Zhang G.G. et al. (2012) (A) and of SHP22-7 (B). Chemical structures of plicacetin (C) and amicetin (D).

box (GTTCACC), resembling the target site of the phosphate control two-component system PhoP/PhoR (Martin and Liras, 2020). This sequence region is also known to be bound by the large size SARP regulator AfsR (Martin and Liras, 2020). Additionally, the central TCA triad can be present in binding motifs recognized by the nitrogen regulator GlnR or the DmdR1 (Flores and Martín, 2004; Martin and Liras, 2020). Cross-talk between different transcriptional regulators via the interaction of the same binding sites have been shown before (Martín et al., 2011; Martin and Liras, 2020). Thus, it is also possible that transcriptional activation of the amicetin BGC is an effect from multiple regulatory interactions.

\section{SARP-Type Regulatory Genes Are Widespread in BGCs From Diverse Actinobacteria}

Of the top 10 genera containing SARP-type regulators in the antiSMASH database 2 (Blin et al., 2019), 9 belong to the phylum Actinobacteria. Broken up by genus, $98 \%$ of Streptomyces (611/625), 81\% of Nocardia (78/96), 100\% of Salinispora (72/72), 100\% of Micromonospora (62/62), and 97\% of Amycolatopsis (38/39) genomes harbor SARP-type regulatory genes, showing their prevalence in filamentous Actinobacteria. On the other hand, only $42 \%$ of Mycobacterium $(115 / 276)$ species have SARP-type regulators. Outside of Actinobacteria, mainly Proteobacteria have hits for the
SMCOG1041 profile, but mostly lack hits for the HTH motif domain or the BTAD (transcriptional activator domain) [e.g., 6\% (79/1236) of Pseudomonas hit the smCoG profile, but none of them contain a BTAD match]. Thus, SMCOG1041 profiles from Proteobacteria may not represent typical SARP-type regulators.

Of the total of 6525 proteins containing a hit against any of the four SARP-related PFAM domains, 3289 (50\%) are from the order Streptomycetales (Figure 8). These again break down into 47\% "small" SARPs (only containing the HTH and BTAD domains) and 36\% "large" SARPs (also containing the NB-ARC and/or TPR domains). The remaining proteins miss the HTH and/or BTAD domains, likely an artifact of bad sequencing data in published draft genomes. The overrepresentation of SARP-type regulators in Streptomycetales may also be explained by the higher abundance of available genomes in the database. Pseudonocardiales cover $17 \%$ of the SARP-type proteins (1080/6525). 31\% (330/1080) are "small" SARPs and 39\% (421/1080) "large" SARPs. In Micromonosporales, accounting for $13 \%(822 / 6526)$ of the dataset, 30\% (244/822) are "small" SARPs, and 40\% (330/822) are "large" SARPs (Figure 8). Interestingly, Micromonosporales is the only order to contain a significant amount $(22 \%, 184 / 882)$ of SARP-type proteins only containing a hit against BTAD without hitting the HTH domain, suggesting a different subfamily of transcriptional activator. Furthermore, SARP genes were found to be present in various different types of BGCs with a prominent abundance in NRPS 

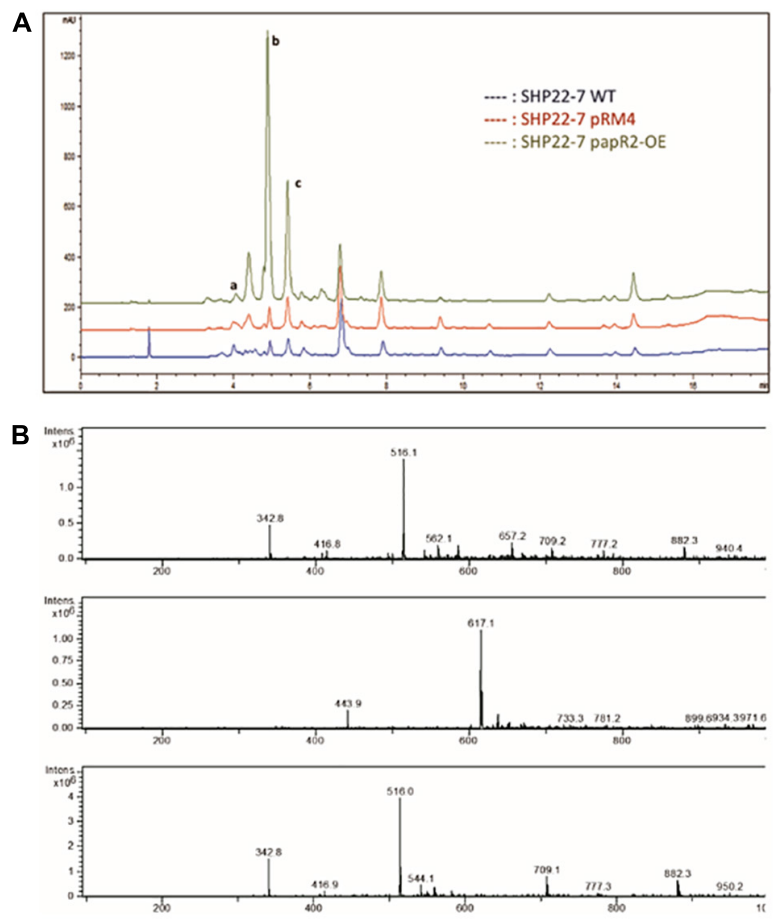

C

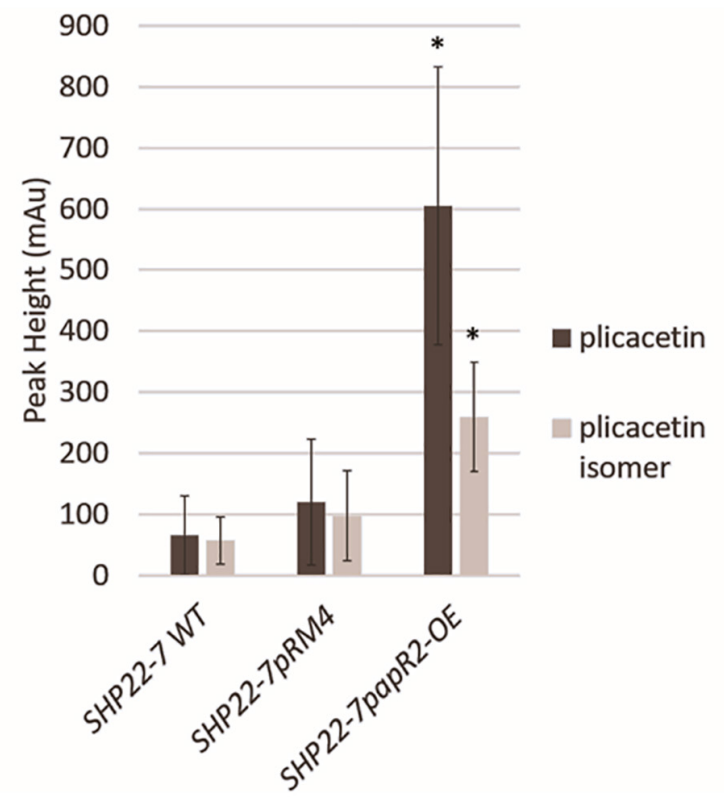

FIGURE 7 | Plicacetin and plicacetin isomer production in the strains SHP22-7 WT, SHP22-7pRM4, and SHP22-7papR2-OE, respectively. HPLC profile of all three strains. Peaks in the HPLC spectrum representing amicetin are marked with $a$, plicacetin marked with $b$, and plicacetin isomer marked with c (A). Mass spectra of plicacetin (Upper), amicetin (Middle), and a plicacetin isomer (Lower) (B) isolated from SHP22-7 WT. Graphical representation of production levels of plicacetin and plicacetin isomer in each strain. $n=10 ;{ }^{*}$ means significance (C).

and PKS gene clusters (Figure 9), which however might also be associated with the higher frequency of these cluster types in the database.

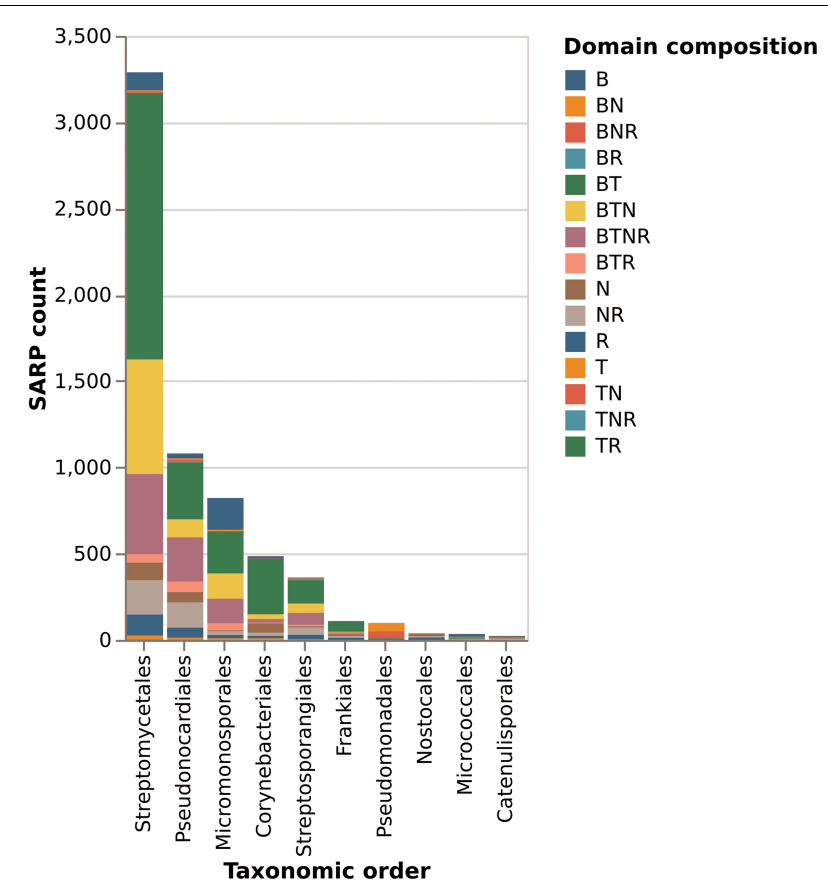

FIGURE 8 | Domain composition of SARP-like proteins in the 10 orders with most protein hits. Letters in the legend denote which domains are present. $B$ is the BTAD domain, $\mathrm{T}$ is the $\mathrm{HTH}$-motif DNA binding domain, $\mathrm{N}$ is the NB-ARC domain of unknown function, and $R$ is the tetratricopeptide repeat domain.

\section{DISCUSSION}

Regulation is the screw plug to unlock the biosynthetic potential of natural compound producers. Here, we showed that heterologous expression of the SARP-type regulatory gene papR2 in the foreign host $S$. lividans leads to transcriptional activation of the silent Red BGC. Since SARP regulators show a comparable protein architecture and bind to similar recognition sequences at the DNA, they can substitute for their regulatory functions as exemplified for PapR2 and RedD. This is also underpinned by a previous study, where it has been shown that overexpression of the SARP gene $v l m l$ from the valanimycin producer Streptomyces viridifaciens in a redD mutant of $S$. coelicolor M512 restores Red production, demonstrating that $v \operatorname{lmI}$ can complement a redD mutation (Garg and Parry, 2010). Besides having gained indications for such kind of cross-regulation, our data also contribute to a better understanding of the regulation of Red biosynthesis in general. So far, there have been only bioinformatic predictions on potential regulatory binding regions within the Red BGC (Iqbal et al., 2012). Here, we provide first experimental evidence by EMSA studies and (q)RT-PCR experiments for the SARP-binding capability to promoter regions (PredP, PredQ) of the Red BGC. These data clearly show that redP and redQ are direct targets of a SARP-driven regulation during Red biosynthesis.

In the literature SARPs often are designated as "pathwayspecific" transcriptional activators, which means that they control 


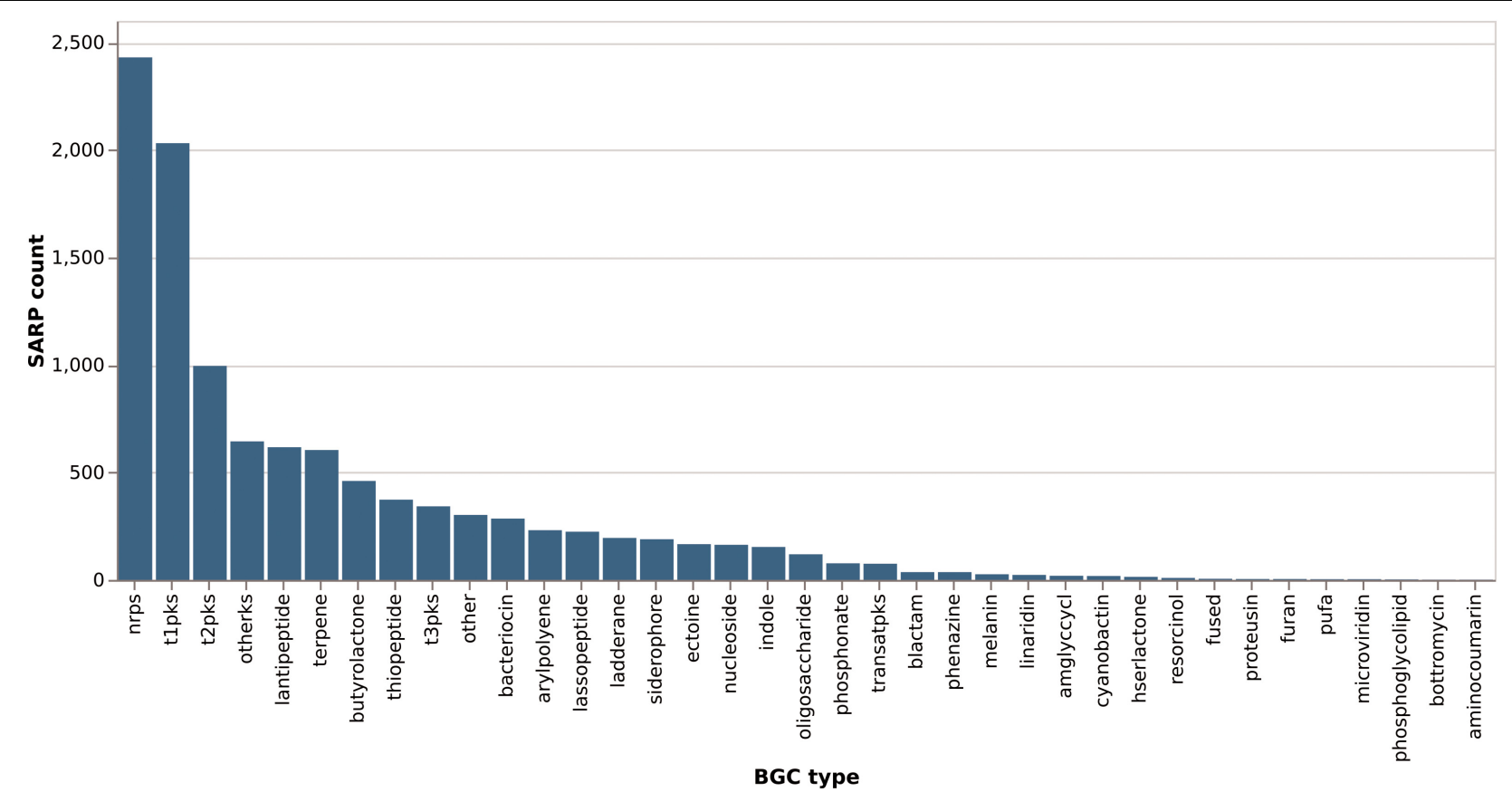

FIGURE 9 | Distribution of SARP-like genes across different biosynthetic gene cluster types. SARP-like genes in hybrid clusters are counted once per type they occur in (e.g., a SARP in a NRPS/PKS type I hybrid cluster would count for both "nrps" and "t1pks").

the expression of an individual BCG. However, SARPs indeed can have activating effects on the biosynthesis of different substances. One example occurring in nature is provided by the SARPtype regulator CcaR from S. clavuligerus, which activates the cephamycin gene cluster, where $c c a R$ is part of, as well as the adjacent clavulanic acid gene cluster (Pérez-Llarena et al., 1997). Thus, the term "pathway-specific" is not accurate for SARPs and should better be replaced by "cluster-situated" as suggested previously (Huang et al., 2005; Liu et al., 2013). Our results show that SARPs have the potential to control different BGCs, when present in different producer organisms: The SARP regulator PapR2 activates the transcription of the corresponding pristinamycin gene cluster in S. pristinaespiralis (Mast et al., 2015) but it also affects different antibiotic BGCs, such as Red and plicacetin, when expressed in foreign strains, such as S. lividans or SHP22-7, respectively. Especially, in SHP22-7 papR2 expression significantly improved plicacetin production and as a result also led to an improved production of a so far not further characterized plicacetin derivative. This derivative is dissimilar from the plicacetin isomer since it shows a different retention time in HPLC analysis ( $6.5 \mathrm{~min}$ ) (Figure 9) and a smaller mass of $481 \mathrm{~m} / z$ (data not shown). If PapR2-driven plicacetin gene cluster activation is a direct or an indirect regulatory effect is unclear at the moment since no direct interaction of the PapR2 regulator with the plicacetin gene cluster could be demonstrated. Furthermore, no SARP gene is present in the plicacetin gene cluster. However, SARP genes have been found in four other SHP22-7 BGCs, which would allow the possibility that one of these regulators may act as the natural activator of amicetin/plicacetin biosynthesis. A similar untargeted effect has been observed when the PAS-LuxR-type regulator PimM from S. natalensis was expressed in S. clavuligerus, which led to an improved production of cephamycin, clavulanic acid, and tunicamycins (Martínez-Burgo et al., 2019). For tunicamycin production the regulatory effect upon pimM expression is unclear. pimM expression improved tunicamycin production without affecting tunicamycin gene cluster transcription. Besides, no pathway-specific activator has been identified within the tunicamycin BGC. In this study, the authors speculated that PimM may exert its effect on tunicamycin production, e.g., due to a positive influence on precursor supply (Martínez-Burgo et al., 2019). Furthermore, they found that PimM from S. natalensis shows some similarity to a PimM-like regulator, encoded by a gene of the S. clavuligerus genome (Martínez-Burgo et al., 2019). Thus, also in these analyses the non-native PimM regulator may have occupied the regulatory role of a homologous natural regulator and along this path provoked antibiotic production. Interspecies cross-regulation has also been shown for the LuxRfamily type (LAL) regulator PikD. In Streptomyces. venezuelae $\mathrm{PikD}$ regulates the expression of pikromycin. The heterologous expression of the $p i k D$ homologous genes rapH and $f k b N$ from Streptomyces hygroscopicus in S. venezuelae increased the production of the antibiotic pikromycin. LAL-family regulators resemble SARP-type regulators in the ATP-binding motif at the $\mathrm{N}$-terminus and in the DNA-binding motif to some extent, which in both regulator types consists of an HTH-motif (Mo and Yoon, 2016).

The fact that SARP genes are widely distributed among actinomycetes, where they occur in many different types of secondary metabolite gene clusters makes them good candidates 
to be used as engineering tools for the activation of BCGs. Especially in Streptomyces they are predominant occurring with a $\sim 100 \%$ abundance. Interestingly, there might be a phylogenetic grouping of different SARP regulator types from different actinobacterial genera. If so this would raise the question if there is a co-evolution of certain regulator genes with their corresponding gene clusters. Besides that, the statistical analysis revealed that there is a different abundance of different types of SARP regulators, belonging either to the small SARPs $(<400$ amino acids) with only HTH and BTAD domains (e.g., ActIIORF4 or RedD) or the large SARPs, which contain an additional NTPase domain and/or a conserved C-terminal TPR domain of unknown function ( $\sim 1000$ residues) (Liu et al., 2013). Overall small SARP type regulators are more abundant in actinomycetes than the large ones (Figure 8). Besides, more experimental data are available on small SARP-guided regulations, and the unclear function of the additional domains included in the large SARPs likely causes further constraints in the function of the regulatory activity. Thus, we propose that especially representatives from the group of small SARP regulators are good candidates to be used for activation approaches. As outlined above, SARP regulators can bind to recognition sequences, which occur at various positions within the promoter of the target genes. The variety of binding sites may also reflect the diversity of SARP-type activators. Thus, it would be interesting to bioinformatically group the SARP regulators by taking into account their DNA-binding domains, which may provide a better picture of the different SARP subsets.

That SARP expression can lead to the activation of silent gene cluster expression has been shown in a recent study. Here, the aim was to activate some of the more than 20 cryptic gene clusters from Streptomyces sp. MSC090213JE08 (Du et al., 2016). In this study the authors combined an OSMAC approach with the expression of several native SARP genes from Streptomyces sp. MSC090213JE08. Thereby, four of the seven generated recombinant SARP expression strains produced nine metabolites that were hardly detected in the control strains. Expression of one of the SARP genes (SARP-7) in Streptomyces sp. MSC090213JE08 led to the production of the novel polyene-like substance ishigamide (Du et al., 2016). This study successfully showed the potency of a SARP-guided silent gene cluster activation. However, the drawback of this approach is that several conditions (number of different culture media) need to be tested and a set of genetic manipulations (cloning of each individual SARP gene) has to be done in order to provoke cluster activation. Further experimental setup is then linked to untargeted laborious compound purifications and analytics. Indeed, this is also the main problem of several other efforts to activate secondary metabolite synthesis in actinomycetes as outlined above. Thus, based on our gained knowledge we propose a targeted SARP-guided strategy for the activation of BGCs in actinomycetes. Our strategy involves (1) prioritization of strains with SARP genes and SARP binding motifs in the BGC. In our experimental setup we focus on SARP genes that encode for predicted proteins with high similarity to PapR2 (>55\% amino acid sequence similarity), as well as the occurrence of a PapR2 consensus motif within the promoter region(s) of the SARPgene containing BGCs, (2) introduction of a SARP-expression construct [in our approach this means heterologous expression of the PapR2 regulator with the help of the pGM190/papR2 and/or pRM4/papR2 expression construct(s)], (3) comparative biological and chemical analyses of SARP-activated expression samples with samples from non-manipulated strains. In our study we focus on PapR2 as the activator brick, however, of course any other type of SARP regulator with a known consensus sequence might be used as the basis for such an activation approach. For sure there might be limitations in such an activation strategy, e.g., transcriptional activation may fail due to SARP-specificity reasons or a lack of a broader set of well-characterized SARP regulators to be tested as candidate elicitors. However, here we disclose a screening idea or a kind of dragnet investigation, which does not aim to cover all possible SARP-regulated clusters but highlights the most probable ones to be activated upon SARP expression. Our strategy has two main advantages: (1) In contrast to completely unspecific cluster activation efforts, such as addition of general elicitors, co-culture approaches or the OSMAC strategy, our approach is more targeted, as it focuses only on a defined set of BGCs, namely those ones that are bioinformatically predicted to be under SARP control. Applying combinatory bioinformatics, such as antiSMASH and PatScan allows to directly identify the associated BGC. In addition, the detection of the activated compound is more straightforward if the outline of the structure can be deduced from the cluster sequence. (2) In contrast to activation strategies that are absolutely specific for the respective BGC, e.g., heterologous expression of the BGC, introduction of artificial promoters in front of the BGC, or the manipulation of clustersituated regulators, no major effort to manipulate the genome is necessary with our procedure since it only involves cloning of one SARP gene-containing expression construct into the respective strain(s) of interest. These major benefits make the activation of BGCs by SARPs a promising strategy to be applied on putative antibiotic producers.

\section{DATA AVAILABILITY STATEMENT}

The complete genome sequence of S. lividans T7 has been deposited at DDBJ/ENA/GenBank under the accession number ACEY00000000. The main genome scaffold sequence of SHP22-7 has been deposited at DDBJ/ENA/GenBank under the accession number QXMM00000000. Raw sequencing data are available under SRA accession number PRJNA489221.

\section{AUTHOR CONTRIBUTIONS}

YM generated the strains SLPGM190 and SLPapR2-OE, carried out the bioinformatic analyses, and designed, supervised, and coordinated the study. KB performed the statistical analyses. JK created the vector $\mathrm{pRM} 4 / \mathrm{papR} 2$ while the vector was inserted in SHP22-7 by IH. IH tested the bioactivity. JK performed all qualitative and quantitative transcriptional analyses. $\mathrm{AK}$ and $\mathrm{IH}$ performed the HPLC analyses. YM, JK, IH, and $\mathrm{KB}$ wrote the manuscript. 


\section{FUNDING}

We gratefully acknowledge the funding received from the BMBF German-Indonesian Cooperation project NAbaUnAk. The study was supported by a DAAD scholarship given to JK and a scholarship from the Indonesian Ministry for Research and Technology for IH.

\section{ACKNOWLEDGMENTS}

We thank Vera Kübler for performing the EMSAs. We also thank Regina Ort-Winklbauer from the University of Tübingen for

\section{REFERENCES}

Aigle, B., Pang, X., Decaris, B., and Leblond, P. (2005). Involvement of AlpV, a new member of the Streptomyces antibiotic regulatory protein family, in regulation of the duplicated type II polyketide synthase alp gene cluster in Streptomyces ambofaciens. J. Bacteriol. 187, 2491-2500. doi: 10.1128/JB.187.7. 2491-2500.2005

Arias, P., Fernández-Moreno, M. A., and Malpartida, F. (1999). Characterization of the pathway-specific positive transcriptional regulator for actinorhodin biosynthesis in Streptomyces coelicolor A3(2) as a DNA-binding protein. J. Bacteriol. 181, 6958-6968. doi: 10.1128/jb.181.22.6958-6968.1999

Baltz, R. H. (2017). Gifted microbes for genome mining and natural product discovery. J. Ind. Microbiol. Biotechnol. 44, 573-588. doi: 10.1007/s10295-016$1815-\mathrm{x}$

Bate, N., Stratigopoulos, G., and Cundliffe, E. (2002). Differential roles of two SARP-encoding regulatory genes during tylosin biosynthesis. Mol. Microbiol. 43, 449-458. doi: 10.1046/j.1365-2958.2002.02756.x

Bibb, M. J. (2005). Regulation of secondary metabolism in streptomycetes. Curr. Opin. Microbiol. 8, 208-215. doi: 10.1016/j.mib.2005.02.016

Blin, K., Pascal Andreu, V., de los Santos, E. L. C., Del Carratore, F., Lee, S. Y., Medema, M. H., et al. (2019). The antiSMASH database version 2: a comprehensive resource on secondary metabolite biosynthetic gene clusters. Nucleic Acids Res. 47, D625-D630. doi: 10.1093/nar/gky1060

Blin, K., Wohlleben, W., and Weber, T. (2018). Patscanui: an intuitive web interface for searching patterns in DNA and protein data. Nucleic Acids Res. 46, W205W208. doi: 10.1093/nar/gky321

Chen, Y., Wendt-Pienkowski, E., and Shen, B. (2008). Identification and utility of FdmR1 as a Streptomyces antibiotic regulatory protein activator for fredericamycin production in Streptomyces griseus ATCC 49344 and heterologous hosts. J. Bacteriol. 190, 5587-5596. doi: 10.1128/JB.00592-08

Du, D., Katsuyama, Y., Onaka, H., Fujie, M., Satoh, N., Shin-ya, K., et al. (2016). Production of a novel amide-containing polyene by activating a cryptic biosynthetic gene cluster in Streptomyces sp. MSC090213JE08. ChemBioChem 17, 1464-1471. doi: 10.1002/cbic.201600167

Fischer, J. (1996). Entwicklung Eines Regulierbaren Expressionssystems zur Effizienten Synthese Rekombinanter Proteine in Streptomyces Lividans. Stuttgart: University of Stuttgart. Ph.D. thesis.

Flores, F. J., and Martín, J. F. (2004). Iron-regulatory proteins DmdR1 and DmdR2 of Streptomyces coelicolor form two different DNA-protein complexes with iron boxes. Biochem. J. 380, 497-503. doi: 10.1042/BJ20031945

Garg, R. P., Ma, Y., Hoyt, J. C., and Parry, R. J. (2002). Molecular characterization and analysis of the biosynthetic gene cluster for the azoxy antibiotic valanimycin. Mol. Microbiol. 46, 505-517. doi: 10.1046/j.1365-2958.2002.03 169. $\mathrm{x}$

Garg, R. P., and Parry, R. J. (2010). Regulation of valanimycin biosynthesis in Streptomyces viridifaciens: characterization of VlmI as a Streptomyces antibiotic regulatory protein (SARP). Microbiology 156, 472-483. doi: 10.1099/mic.0. 033167-0

Handayani, I., Ratnakomala, S., Lisdiyanti, P., Fahrurrozi Kusharyoto, W., Alanjary, M., et al. (2018). Complete genome sequence of Streptomyces excellent technical assistance. We acknowledge help from Harald Gross, Nico Ortlieb, and Dorothee Wistuba from the University of Tübingen for High-Resolution Mass Spectrometric analysis. Finally, we thank Puspita Lisdiyanti and Shanti Ratna Komala from the Indonesian Institute of Sciences for providing us with the Indonesian strain isolate SHP 22-7.

\section{SUPPLEMENTARY MATERIAL}

The Supplementary Material for this article can be found online at: https://www.frontiersin.org/articles/10.3389/fmicb. 2020.00225/full\#supplementary-material

sp. strain SHP22-7, a new species isolated from mangrove of Enggano island. Indonesia. Microbiol. Resour. Announc. 7, 1-3. doi: 10.1128/MRA.013 17-18

He, X., Li, R., Pan, Y., Liu, G., and Tan, H. (2010). SanG, a transcriptional activator, controls nikkomycin biosynthesis through binding to the sanN-sanO intergenic region in Streptomyces ansochromogenes. Microbiology 156, 828-837. doi: 10. 1099/mic.0.033605-0

Horinouchi, S., Suzuki, H., and Beppu, T. (1986). Nucleotide sequence of afsB, a pleiotropic gene involved in secondary metabolism in Streptomyces coelicolor A3(2) and Streptomyces lividans. J. Bacteriol. 168, 257-269. doi: 10.1128/JB.168. 1.257-269.1986

$\mathrm{Hu}, \mathrm{H}$., Zhang, Q., and Ochi, K. (2002). Activation of antibiotic biosynthesis by specified mutations in the $r p o B$ gene (encoding the RNA polymerase $\beta$ subunit) of Streptomyces lividans. J. Bacteriol. 184, 3984-3991. doi: 10.1128/JB.184.14. 3984-3991.2002

Huang, J., Shi, J., Molle, V., Sohlberg, B., Weaver, D., Bibb, M. J., et al. (2005). Crossregulation among disparate antibiotic biosynthetic pathways of Streptomyces coelicolor. Mol. Microbiol. 58, 1276-1287. doi: 10.1111/j.1365-2958.2005.04 879.x

Iqbal, M., Mast, Y., Amin, R., Hodgson, D. A., Stream Consortium, Wohlleben, W., et al. (2012). Extracting regulator activity profiles by integration of de novo motifs and expression data: characterizing key regulators of nutrient depletion responses in Streptomyces coelicolor. Nucleic Acids Res. 40, 5227-5239. doi: 10.1093/nar/gks205

Kieser, T., Bibb, M. J., Buttner, M. J., Chater, K. F., and Hopwood, D. A. (2000). Practical Streptomyces genetics. John Innes Found. Norwich, Engl. 3, 260-261. doi: 10.2436/im.v3i4.9291

Korzybski, T., Kowszyk-Gindifer, Z., Kuryłowicz, W., and Paryski, E. (2013). Antibiotics. Volume 1: Origin, Nature and Properties. Oxford: Pergamon Press.

Kurniawan, Y. N., Kitani, S., Maeda, A., and Nihira, T. (2014). Differential contributions of two SARP family regulatory genes to indigoidine biosynthesis in Streptomyces lavendulae FRI-5. Appl. Microbiol. Biotechnol. 98, 9713-9721. doi: 10.1007/s00253-014-5988-9

Lewis, R. A., Laing, E., Allenby, N., Bucca, G., Brenner, V., Harrison, M., et al. (2010). Metabolic and evolutionary insights into the closely-related species Streptomyces coelicolor and Streptomyces lividans deduced from high-resolution comparative genomic hybridization. BMC Genomics 11:682. doi: 10.1186/14712164-11-682

Liu, G., Chater, K. F., Chandra, G., Niu, G., and Tan, H. (2013). Molecular regulation of antibiotic biosynthesis in Streptomyces. Microbiol. Mol. Biol. Rev. 77, 112-143. doi: 10.1128/MMBR.00054-12

Livak, K. J., and Schmittgen, T. D. (2001). Analysis of relative gene expression data using real-time quantitative PCR and the 2- $\Delta \Delta$ CT method. Methods 25, 402-408. doi: 10.1006/METH.2001.1262

Lombó, F., Braña, A. F., Méndez, C., and Salas, J. A. (1999). The mithramycin gene cluster of Streptomyces argillaceus contains a positive regulatory gene and two repeated DNA sequences that are located at both ends of the cluster. J. Bacteriol. 181, 642-647. doi: 10.1128/jb.181.2.642-647.1999

Martin, J., and Liras, P. (2020). The balance metabolism safety net: integration of stress signals by interacting transcriptional factors in Streptomyces and related 
Actinobacteria. Front. Microbiol. accepted 10:3120. doi: 10.3389/fmicb.2019. 03120

Martín, J. F., Sola-Landa, A., Santos-Beneit, F., Fernández-Martínez, L. T., Prieto, C., and Rodríguez-García, A. (2011). Cross-talk of global nutritional regulators in the control of primary and secondary metabolism in Streptomyces. Microb. Biotechnol. 4, 165-174. doi: 10.1111/j.1751-7915.2010.00235.x

Martinez, A., Kolvek, S. J., Hopke, J., Yip, C. L. T., and Osburne, M. S. (2005). Environmental DNA fragment conferring early and increased sporulation and antibiotic production in Streptomyces species. Appl. Environ. Microbiol. 71, 1638-1641. doi: 10.1128/AEM.71.3.1638-1641.2005

Martínez-Burgo, Y., Santos-Aberturas, J., Rodríguez-García, A., Barreales, E. G., Tormo, J. R., Truman, A. W., et al. (2019). Activation of secondary metabolite gene clusters in Streptomyces clavuligerus by the PimM regulator of Streptomyces natalensis. Front. Microbiol. 10:580. doi: 10.3389/fmicb.2019. 00580

Mast, Y., Guezguez, J., Handel, F., and Schinko, E. (2015). A complex signaling cascade governs pristinamycin biosynthesis in Streptomyces pristinaespiralis. Appl. Environ. Microbiol. 81, 6621-6636. doi: 10.1128/AEM.00728-15

Medema, M. H., Blin, K., Cimermancic, P., de Jager, V., Zakrzewski, P., Fischbach, M. A., et al. (2011). antiSMASH: rapid identification, annotation and analysis of secondary metabolite biosynthesis gene clusters in bacterial and fungal genome sequences. Nucleic Acids Res. 39, W339-W346. doi: 10.1093/nar/gkr466

Mo, S., Sydor, P. K., Corre, C., Alhamadsheh, M. M., Stanley, A. E., Haynes, S. W., et al. (2008). Elucidation of the Streptomyces coelicolor pathway to 2undecylpyrrole, a key intermediate in undecylprodiginine and streptorubin B biosynthesis. Chem. Biol. 15, 137-148. doi: 10.1016/j.chembiol.2007.11.015

Mo, S., and Yoon, Y. J. (2016). Interspecies complementation of the LuxR family pathway-specific regulator involved in macrolide biosynthesis. J. Microbiol. Biotechnol. 26, 66-71. doi: 10.4014/jmb.1510.10085

Newman, D. J., and Cragg, G. M. (2016). Natural products as sources of new drugs from 1981 to 2014. J. Nat. Prod. 79, 629-661. doi: 10.1021/acs.jnatprod.5b0 1055

Novakova, R., Rehakova, A., Kutas, P., Feckova, L., and Kormanec, J. (2011). The role of two SARP family transcriptional regulators in regulation of the auricin gene cluster in Streptomyces aureofaciens CCM 3239. Microbiology 157, 1629-1639. doi: 10.1099/mic.0.047795-0

Ochi, K., and Hosaka, T. (2013). New strategies for drug discovery: activation of silent or weakly expressed microbial gene clusters. Appl. Microbiol. Biotechnol. 97, 87-98. doi: 10.1007/s00253-012-4551-9

Onaka, H., Mori, Y., Igarashi, Y., and Furumai, T. (2011). Mycolic acid-containing bacteria induce natural-product biosynthesis in Streptomyces species. Appl. Environ. Microbiol. 77, 400-406. doi: 10.1128/AEM.01337-10

Onaka, H., Nakagawa, T., and Horinouchi, S. (2002). Involvement of two A-factor receptor homologues in Streptomyces coelicolor A3(2) in the regulation of secondary metabolism and morphogenesis. Mol. Microbiol. 28, 743-753. doi: 10.1046/j.1365-2958.1998.00832.x

Pérez-Llarena, F. J., Liras, P., Rodríguez-García, A., and Martín, J. F. (1997). A regulatory gene $(c c a R)$ required for cephamycin and clavulanic acid production in Streptomyces clavuligerus: amplification results in overproduction of both beta-lactam compounds. J. Bacteriol. 179, 2053-2059. doi: 10.1128/jb.179.6. 2053-2059.1997

Pulsawat, N., Kitani, S., Fukushima, E., and Nihira, T. (2009). Hierarchical control of virginiamycin production in Streptomyces virginiae by three pathway-specific regulators: VmsS. VmsT and VmsR. Microbiology 155, 1250-1259. doi: 10.1099/ mic.0.022467-0

Rehakova, A., Novakova, R., Feckova, L., Mingyar, E., and Kormanec, J. (2013). A gene determining a new member of the SARP family contributes to transcription of genes for the synthesis of the angucycline polyketide auricin in Streptomyces aureofaciens CCM 3239. FEMS Microbiol. Lett. 346, 45-55. doi: 10.1111/1574-6968.12200

Rodríguez, H., Rico, S., Díaz, M., and Santamaría, R. I. (2013). Two-component systems in Streptomyces: key regulators of antibiotic complex pathways. Microb. Cell Fact. 12:127. doi: 10.1186/1475-2859-12-127

Rodríguez, M., Núñez, L. E., Braña, A. F., Méndez, C., Salas, J. A., and Blanco, G. (2008). Identification of transcriptional activators for thienamycin and cephamycin C biosynthetic genes within the thienamycin gene cluster from Streptomyces cattleya. Mol. Microbiol. 69, 633-645. doi: 10.1111/j.1365-2958. 2008.06312.x
Ryding, N. J., Anderson, T. B., and Champness, W. C. (2002). Regulation of the Streptomyces coelicolor calcium-dependent antibiotic by absA, encoding a cluster-linked two-component system. J. Bacteriol. 184, 794-805. doi: 10.1128/ jb.184.3.794-805.2002

Salehi-Najafabadi, Z., Barreiro, C., Rodríguez-García, A., Cruz, A., López, G. E., and Martín, J. F. (2014). The gamma-butyrolactone receptors BulR1 and BulR2 of Streptomyces tsukubaensis: tacrolimus (FK506) and butyrolactone synthetases production control. Appl. Microbiol. Biotechnol. 98, 4919-4936. doi: 10.1007/s00253-014-5595-9

Sambrook, J., Fritsch, E. F., and Maniatis, T. (1989). Molecular Cloning: a Laboratory Manual. Berlin: Cold Spring Harbor Laboratory Press.

Santamarta, I., Rodríguez-García, A., Pérez-Redondo, R., Martín, J. F., and Liras, P. (2002). CcaR is an autoregulatory protein that binds to the $c c a R$ and $c e f D-$ cmcI promoters of the cephamycin C-clavulanic acid cluster in Streptomyces clavuligerus. J. Bacteriol. 184, 3106-3113. doi: 10.1128/JB.184.11.3106-3113. 2002

Sheldon, P. J., Busarow, S. B., and Hutchinson, C. R. (2002). Mapping the DNA-binding domain and target sequences of the Streptomyces peucetius daunorubicin biosynthesis regulatory protein. DnrI. Mol. Microbiol. 44, 449460. doi: 10.1046/j.1365-2958.2002.02886.x

Suzuki, T., Mochizuki, S., Yamamoto, S., Arakawa, K., and Kinashi, H. (2010). Regulation of lankamycin biosynthesis in Streptomyces rochei by two SARP genes, srrY and srrZ. Biosci. Biotechnol. Biochem. 74, 819-827. doi: 10.1271/bbb. 90927

Takano, E., Gramajo, H. C., Strauch, E., Andres, N., White, J., and Bibb, M. J. (1992). Transcriptional regulation of the RedD transcriptional activator gene accounts for growth-phase-dependent production of the antibiotic undecylprodigiosin in Streptomyces coelicolor A3(2). Mol. Microbiol. 6, 27972804. doi: 10.1111/j.1365-2958.1992.tb01459.x

Takano, E., Kinoshita, H., Mersinias, V., Bucca, G., Hotchkiss, G., Nihira, T., et al. (2005). A bacterial hormone (the SCB1) directly controls the expression of a pathway-specific regulatory gene in the cryptic type I polyketide biosynthetic gene cluster of Streptomyces coelicolor. Mol. Microbiol. 56, 465-479. doi: 10. $1111 /$ j.1365-2958.2005.04543.x

Tanaka, A., Takano, Y., Ohnishi, Y., and Horinouchi, S. (2007). AfsR recruits RNA polymerase to the afs $S$ promoter: a model for transcriptional activation by SARPs. J. Mol. Biol. 369, 322-333. doi: 10.1016/J.JMB.2007.02.096

Tanaka, Y., and Omura, S. (1990). Metabolism and products of actinomycetes. An introduction. Actinomycetologica 4, 13-14. doi: 10.3209/saj.4_13

United Nations Foundation and the Wellcome Charitable Trust, (2016). Review on Antimicrobial Resistance. Available at: https://amr-review.org/ (Accessed September 6, 2018).

van Wezel, G. P., White, J., Hoogvliet, G., and Bibb, M. J. (2000). Application of redD, the transcriptional activator gene of the undecylprodigiosin biosynthetic pathway, as a reporter for transcriptional activity in Streptomyces coelicolor A3(2) and Streptomyces lividans. J. Mol. Microbiol. Biotechnol. 2, 551-556.

White, J., and Bibb, M. (1997). bldA dependence of undecylprodigiosin production in Streptomyces coelicolor A3(2) involves a pathway-specific regulatory cascade. J. Bacteriol. 179, 627-633. doi: 10.1128/JB.179.3.627-633.1997

Widdick, D. A., Dodd, H. M., Barraille, P., White, J., Stein, T. H., Chater, K. F., et al. (2003). Cloning and engineering of the cinnamycin biosynthetic gene cluster from Streptomyces cinnamoneus cinnamoneus DSM 40005. Proc. Natl. Acad. Sci. U.S.A. 100, 4316-4321. doi: 10.1073/pnas.0230516100

Wietzorrek, A., and Bibb, M. (1997). A novel family of proteins that regulates antibiotic production in streptomycetes appears to contain an OmpR-like DNAbinding fold. Mol. Microbiol. 25, 1181-1184. doi: 10.1046/j.1365-2958.1997. 5421903.x

World Health Organization, (2015). Global Action plan on Antimicrobial Resistance. Available at: http://www.who.int/antimicrobial-resistance/ publications/global-action-plan/en/ (Accessed September 6, 2018).

Worldbank, (2016). Drug-Resistant Infections: A Threat to our Economic Future. Available at: http://www.worldbank.org/en/news/press-release/2016/09/18/by2050-drug-resistant-infections-could-cause-global-economic-damage- onpar-with-2008-financial-crisis (Accessed September 6, 2018).

Wu, X., Jin, L., Zhang, H., Tong, R., Ma, M., and Chen, Y. (2018). Identification of truncated form of NosP as a transcription factor to regulate the biosynthesis of nosiheptide. FASEB J. 32, 453-465. doi: 10.1096/fj.201700556R 
Xie, Y., Wang, B., Liu, J., Zhou, J., Ma, J., Huang, H., et al. (2012). Identification of the biosynthetic gene cluster and regulatory cascade for the synergistic antibacterial antibiotics griseoviridin and viridogrisein in Streptomyces griseoviridis. ChemBioChem 13, 2745-2757. doi: 10.1002/cbic. 201200584

Ye, S., Braña, A. F., González-Sabín, J., Morís, F., Olano, C., Salas, J. A., et al. (2018). New insights into the biosynthesis pathway of polyketide alkaloid argimycins $\mathrm{P}$ in Streptomyces argillaceus. Front. Microbiol. 9:252. doi: 10.3389/fmicb.2018. 00252

Zhang, G. G., Zhang, H., Li, S., Xiao, J., Zhang, G. G., Zhu, Y., et al. (2012). Characterization of the amicetin biosynthesis gene cluster from Streptomyces vinaceusdrappus NRRL 2363 implicates two alternative strategies for amide bond formation. Appl. Environ. Microbiol. 78, 2393-2401. doi: 10.1128/AEM. 07185- 11

Zhang, X., Davenport, K. W., Gu, W., Daligault, H. E., Munk, A. C., Tashima, H., et al. (2012). Improving genome assemblies by sequencing PCR products with PacBio. Biotechniques 53, 61-62. doi: 10.2144/0000113891
Zhu, H., Sandiford, S. K., and van Wezel, G. P. (2014). Triggers and cues that activate antibiotic production by actinomycetes. J. Ind. Microbiol. Biotechnol. 41, 371-386. doi: 10.1007/s10295-013-1309-z

Conflict of Interest: The authors declare that the research was conducted in the absence of any commercial or financial relationships that could be construed as a potential conflict of interest.

The reviewer JA declared a past co-authorship with one of the authors AK to the handling Editor.

Copyright (c) 2020 Krause, Handayani, Blin, Kulik and Mast. This is an open-access article distributed under the terms of the Creative Commons Attribution License (CC BY). The use, distribution or reproduction in other forums is permitted, provided the original author(s) and the copyright owner(s) are credited and that the original publication in this journal is cited, in accordance with accepted academic practice. No use, distribution or reproduction is permitted which does not comply with these terms. 